\title{
Analysis and FPGA Realization of a Novel 5D Hyperchaotic Four-Wing Memristive System, Active Control Synchronization, and Secure Communication Application
}

\author{
Fei Yu $\mathbb{D}^{1},{ }^{1}$ Li Liu $\mathbb{D}^{1},{ }^{1}$ Binyong He $\mathbb{D}^{1},{ }^{1}$ Yuanyuan Huang $\mathbb{D}^{1},{ }^{1}$ Changqiong Shi, ${ }^{1}$ Shuo Cai $\mathbb{D}^{1},{ }^{1}$ \\ Yun Song $\mathbb{D}^{1},{ }^{1}$ Sichun Du $\mathbb{D}^{2},{ }^{2}$ and Qiuzhen Wan $\mathbb{D}^{3}$ \\ ${ }^{1}$ School of Computer and Communication Engineering, Changsha University of Science and Technology, Changsha 410114, China \\ ${ }^{2}$ College of Computer Science and Electronic Engineering, Hunan University, Changsha 410082, China \\ ${ }^{3}$ College of Information Science and Engineering, Hunan Normal University, Changsha 410081, China
}

Correspondence should be addressed to Fei Yu; yufeiyfyf@csust.edu.cn and Yuanyuan Huang; snailhyy@126.com

Received 1 September 2019; Revised 26 October 2019; Accepted 29 October 2019; Published 28 November 2019

Guest Editor: Viet-Thanh Pham Copyright @ 2019 Fei Yu et al. This is an open access article distributed under the Creative Commons Attribution License, which
permits unrestricted use, distribution, and reproduction in any medium, provided the original work is properly cited.

By introducing a flux-controlled memristor with quadratic nonlinearity into a 4D hyperchaotic system as a feedback term, a novel 5D hyperchaotic four-wing memristive system (HFWMS) is derived in this paper. The HFWMS with multiline equilibrium and three positive Lyapunov exponents presented very complex dynamic characteristics, such as the existence of chaos, hyperchaos, limit cycles, and periods. The dynamic characteristics of the HFWMS are analyzed by using equilibria, phase portraits, poincare map, Lyapunov exponential spectrum, bifurcation diagram, and spectral entropy. Of particular interest is that this novel system can generate two-wing hyperchaotic attractor under appropriate parameters and initial conditions. Moreover, the FPGA realization of the novel 5D HFWMS is reported, which prove that the system has complex dynamic behavior. Finally, synchronization of the 5D hyperchaotic system with different structures by active control and a secure signal masking application of the HFWMS are implemented based on numerical simulations and FPGA. This research demonstrates that the hardware-based design of the 5D HFWMS can be applied to various chaos-based embedded system applications including random number generation, cryptography, and secure communication.

\section{Introduction}

Nonlinear science is a new interdisciplinary subject to study the universality of nonlinear phenomena, which runs through almost every subject of meteorology $[1,2]$, mathematics [3-6], fluid mechanics [7, 8], complex network [9-12], electronics [13-15], and social science [16, 17]. Chaos is one of the most important achievements of nonlinear science. Its random-like and sensitive initial values make chaos have good potential applications in the fields of random number generation [18-20], cryptosystem [21, 22], image encryption [23-25] and secure communication [26-29]. In recent years, many new multiwing (or multiscroll) chaotic systems have been discovered and proposed [30-35]. The continuous introduction of various complex chaotic attractor models not only provides research basis for the development of chaotic system theory, but also provides rich subjects for the practical application of chaotic theory.

Since Rossler proposed the first hyperchaotic system with two positive Lyapunov exponents [36], a large number of researchers have begun to devote themselves to the study of hyperchaotic systems [37-42]. Hyperchaotic systems are more sensitive, pseudorandom, and have larger key space, which makes them more suitable for applications such as secure communication and image encryption than chaotic systems. In order to construct more complex chaotic attractors, a large number of literatures have recently reported the multiwing hyperchaotic systems [43-46]. In [43], in order to overcome the inherent difficulties of iteratively adjusting multiparameters in traditional multiparameter control, a unified step function for single-parameter control is proposed to construct a nonequilibrium multiwing 
hyperchaotic system. In [45], by introducing mirror symmetric transformation into the hyperchaotic system, various hyperchaotic attractors with mirror symmetric grids are obtained. In [46], a five-dimensional (5D) autonomous hyperchaotic attractor with four wing is introduced, which has only eight parameters to be controlled and only one equilibrium point.

Memristor is an electronic device that describes the relationship between charge and magnetic flux, which was proposed in 1971 by Chua [47] and for the first time realized by HP Labs in 2008 [48]. Because of its strong nonlinear characteristics, memristors have potential applications in many engineering fields, and their research has attracted more and more attention $[49,50]$. Recently, many nonlinear memristive oscillators have been proposed [51-53], creating a memristive hyperchaotic system with a multiwing attractor having a practical significance, which has become a research hotspot [54-57]. In [55], by introducing a flux-controlled memristor into a multiwing system, a multiwing hyperchaotic attractor is observed in a no-equilibrium memristive system. In [56], a hyperchaotic system is constructed by adding only a smooth flux-controlled memristor to the $3 \mathrm{D}$ pseudo four-wing chaotic system, which can generate a real four-wing hyperchaotic attractor with a line of equilibrium. In [57], flux-controlled memristors are used to replace the resistors in the circuit of the modified Lü system, and this new memristive system can exhibit the hyperchaotic multiwing attractor with two relatively large positive Lyapunov exponents. However, a review of literature revealed there are no research studies that examined the four-wing behavior and three-positive Lyapunov exponents in memristive hyperchaotic systems with dimensions greater than four. This kind of high-dimensional hyperchaotic systems cannot be ignored. Because of their complexity, signal generation is usually used for random number generation and secure communication just to name a few.

In recent years, the main methods to realize chaotic or hyperchaotic attractors are analog circuits, such as breadboards based on discrete components [55-57] and integrated circuits (ICs) based on CMOS technology $[14,33,34,37]$. With the change of time and temperature in analog circuit, the device will have temperature drift and poor control accuracy. Therefore, it is difficult to realize the chaotic system with high precision by analog circuit, and the breadboard is not easy to carry and digitally store. The design of high-dimensional chaotic systems using CMOS technology generally requires multipliers, which are difficult to design. At the same time, ICs have the shortcomings of long development cycle and high cost [58-60]. Therefore, researchers began to focus on digital circuits with low cost, short design cycle, fast speed, low power consumption, and high accuracy, such as digital signal processor (DSP) [61, 62] and field programmable gate array (FPGA) [63-66]. It takes a long time for DSPs to generate chaotic signals at high frequencies and DSP chips to perform operations in order to calculate the value of output signals. On the other hand, FPGA chips have a relatively flexible architecture to achieve parallel operation, and the design and test cycles of the chips is particularly low [67]. In order to increase and expand engineering applications based on chaos, chaotic systems are diversified and need flexible architecture support. With the digitalization and reconfigurability of the FPGA, chaotic systems and their applications can be more flexible. Thus, different forms of signals can be easily generated with the change of parameters of chaotic systems. In addition, the related memristive chaotic system can also be realized alternately by various memristor functions. At present, there are several studies related to designs of chaotic systems based on FPGA. Tuna et al. [63] implemented the Liu-Chen chaotic system on the Xilinx virtex-6 FPGA chip using the 32-bit IQ-Math fixed-point number Heun algorithm. Ahmadi et al. [64] designed a 5D chaotic system on the Xilinx Kintex-7 KC-705 kit FPGA chip using the IEEE 754 32-bit fixed-point number Euler's method. Xu et al. [65] designed a 3D memristive chaotic system on the Xilinx Spartan-6 FPGA chip using the Euler's method of IEEE 754 32-bit floating-point number standard. As far as we know, few literatures have reported the realization of the $5 \mathrm{D}$ memristive hyperchaotic system based on FPGA.

Synchronization of chaotic systems has attracted much attention in recent years due to their applications in chemical reactors, secure communication, and the development of secure cryptosystems [68, 69]. Aiming at chaotic synchronization, several methods such as linear feedback control [70], sliding mode control [71], adaptive control [72-74], backstepping nonlinear control [75], shape control [76, 77], and active control [78-80] have been proposed for synchronization of chaotic systems. Compared with other synchronization methods, the active control method is simple, efficient, and flexible which has been successfully applied to the synchronization of chaotic systems. In [79], the synchronization and antisynchronization of the fractional-order chaotic financial system with market confidence are studied by using the active control method. The results show that the speed of synchronization (antisynchronization) increases with the increase of the order. In [80], the synchronization of chaotic systems with different orders under the influence of unknown model uncertainties and external disturbances is studied by using robust generalized active control approach. With the rapid development of computer technology, more and more attention has been paid to information security [81-91]. Secure communication based on chaotic synchronization is an important branch of information security research, which has been widely studied by many scholars [92-96]. Because of the pseudorandomness, unpredictability, and initial sensitivity of memristive chaotic systems, the encrypted information can be hidden in chaotic signals which are highly similar to the noise. In [97], based on the synchronization of the memristive chaotic system, the encrypting and decrypting of information signals are carried out to realize the secure communication with the help of LabVIEW. However, whether chaotic synchronization is achieved by the active control method [78-80] or secure communication based on chaotic synchronization [92-96], numerical simulations is used to achieve these designed methods. In some chaotic information systems, such as chaotic-based CDMA communications and many other chaotic digital information systems, digital implementation may be required [98-100]. 
Motivated by the above discussions, based on a fluxcontrolled memristor model and the 4D hyperchaotic system introduced in [39], a 5D hyperchaotic system is proposed. Most importantly, the new system generates fourwing and two-wing hyperchaotic attractor phenomenon with three and two positive Lyapunov exponents, respectively and exhibits hyperchaos with multiline equilibrium. Complete dynamic properties of this new system are studied. Also, with the help of FPGA implementation, this $5 \mathrm{D}$ hyperchaotic four-wing memristive system (HFWMS) is realized. Finally, active control synchronization of the 5D hyperchaotic system with different structures and a secure signal masking application of the 5D HFWMS are implemented based on numerical simulations and FPGA.

This paper is organized as follows. In Section 2, the novel 5D HFWMS with multiline equilibrium is introduced and its dynamic properties are discussed. Section 3 is devoted to design, test, and analysis results of FPGA-based HFWMS. In addition, the active control synchronization and chaotic secure communication design of the 5D HFWMS are achieved, and the FPGA experimental results are presented. Section 4 concludes this paper with a summary of the main results.

\section{Novel 5D HFWMS and Its Dynamic Properties}

2.1. The 5D HFWMS. Recently, Volos et al. [39] have announced a novel $4 \mathrm{D}$ four-wing hyperchaotic system, which is described by

$$
\left\{\begin{array}{l}
\dot{x}=a x+y+y z-c w \\
\dot{y}=y z-x z \\
\dot{z}=-z-m x y+b \\
\dot{w}=x
\end{array}\right.
$$

where $a, b, c$, and $m$ are all constants and $x, y, z$, and $w$ are the state variables. System (1) has two positive Lyapunov exponents, showing the four-wing hyperchaotic attractor. Unlike most existing hyperchaotic systems, this hyperchaotic system has a saddle-focus equilibrium and the second equation of the system has no linear term.

In this paper, by introducing a flux-controlled memristor to the first equation of system (1), a novel 5D HFWMS is derived by

$$
\left\{\begin{array}{l}
\dot{x}=a x+d W(\varphi) y+y z-c w \\
\dot{y}=y z-x z \\
\dot{z}=-z-m x y+b \\
\dot{w}=x \\
\dot{\varphi}=y
\end{array}\right.
$$

where $d$ is a positive parameter, expressed as memristor strength and $W(\varphi)$ is a memductance function, defined as $W(\varphi)=d q(\varphi) / d \varphi$. Here, the $\varphi-q$ characteristic curve of the memristor is given by a smooth continuous cubic monotone increasing nonlinearity [46-49], and then the memductance is given by

$$
W(\varphi)=\frac{d q(\varphi)}{d \varphi}=\frac{d\left(e \varphi+n \varphi^{3}\right)}{d \varphi}=e+3 n \varphi^{2},
$$

where $e$ and $n$ are two positive constants. This flux-controlled memristor is easier to analyze and implement. At present, many researchers use the special nonlinear dynamic characteristics of this memristor to construct complex chaotic oscillators [54-57].

2.2. Equilibria and Stability. The equilibria of system (2) are obtained by setting its right-hand side to zero, that is,

$$
\left\{\begin{array}{l}
a x+d\left(e+3 n \varphi^{2}\right) y+y z-c w=0 \\
y z-x z=0 \\
-z-m x y+b=0 \\
x=0 \\
y=0
\end{array}\right.
$$

Through equation (4), we can easily observe that the equilibria of system (2) is a multiline equilibrium point $O=\{(x, y, z, w) \mid x=y=w=0, z=b, \varphi=\eta\}$, where $b$ is an integer and $\eta$ is an arbitrary real constant.

The Jacobian matrix of system (2) at the multiline equilibrium point is

$$
J_{O}=\left[\begin{array}{ccccc}
a & d\left(e+3 n \varphi^{2}\right)+z & y & -c & 6 n \varphi d y \\
-z & z & y-x & 0 & 0 \\
-m y & -m x & -1 & 0 & 0 \\
1 & 0 & 0 & 0 & 0 \\
0 & 1 & 0 & 0 & 0
\end{array}\right]
$$

According to the Jacobian matrix (5), we can obtain the characteristic equation of system (2) as follows:

$$
\lambda(\lambda+1)\left[\lambda^{3}-(a+b) \lambda^{2}+\left(b^{2}+d b e+3 d b n \varphi^{2}+c+a b\right) \lambda-b c\right]=0 .
$$

Equation (6) can be rewritten to as follows:

$$
\lambda(\lambda+1)\left[\lambda^{3}+m 1 \lambda^{2}+m 2 \lambda+m 3\right]=0
$$

where

$$
\left\{\begin{array}{l}
m 1=-(a+b) \\
m 2=\left(b^{2}+d b e+3 d b n \varphi^{2}+c+a b\right) \\
m 3=-b c
\end{array}\right.
$$

From the eigenvalue equation (7), it can be seen that Jacobian matrix (5) has one zero eigenvalue, one negative eigenvalue, and three nonzero eigenvalues. To judge whether system (2) is stable, it is necessary to discriminate the three nonzero eigenvalues. According to the Routh-Hurwitz stability criterion, the need equation (8) satisfies

$$
\left\{\begin{array}{l}
m 1>0 \\
m 3>0 \\
m 1 m 2-m 3>0
\end{array}\right.
$$


If all three conditions in equation (9) are satisfied, the multiline equilibrium point $O$ is stable, otherwise it is unstable. The unstable equilibrium of the system will lead to chaotic behavior. When $a=1, b=1, c=0.7, m=1, d=0.2$, $e=0.1, n=0.01, m 1$, and $m 2$ are both less than zero, so we can judge that system (2) is unstable.

2.3. Symmetry and Dissipativity. The proposed 5D HFWMS has the same symmetry as system (1), and both of them are invariant with respect to $z$-axis symmetry under coordinate transformation $(x, y, z, w, \varphi) \longrightarrow(-x,-y, z,-w,-\varphi)$.

Furthermore, by calculating

$$
\nabla V=\frac{\partial \dot{x}}{\partial x}+\frac{\partial \dot{y}}{\partial y}+\frac{\partial \dot{z}}{\partial z}+\frac{\partial \dot{w}}{\partial w}+\frac{\partial \dot{\varphi}}{\partial \varphi}=a+z-1
$$

when $a+z<1$, system (2) is dissipative and converges exponentially.

2.4. Analysis of the 5D HFWMS. Here, the dynamic behavior of the 5D HFWMS is numerically investigated by the use of several tools such as the phase portraits, poincare map, Lyapunov exponentials, and bifurcation diagram.

2.4.1. Four-Wing Hyperchaotic Attractor. When the system parameters are selected $a=1, b=1, c=0.7, m=1, d=0.2$, $e=0.1$, and $n=0.01$ and the initial condition is set to $[1,-1,1,1,1]$, the phase portraits of system (2) shown in Figure 1 are obtained by Matlab simulation, which is a typical four-wing chaotic attractor, and the time variations of state equations $x, y, z$, and $w$ are provided in Figure 2.

The Lyapunov exponent is used to measure the perturbation caused by initial conditions. If there are slight differences in the system, two adjacent trajectories in the phase space are separated exponentially with time. The Lyapunov exponent is a useful tool for measuring chaotic systems, in particular, it is usually determines whether a chaotic system is chaotic or hyperchaotic according to the number of positive Lyapunov exponents. According to the given system parameters and initial conditions, the Lyapunov exponent of $a \in[-1,1]$ is simulated by the Jacobi matrix method. The numerical results are shown in Figure 3(a) (the five LE5 is out of plot). From Figure 3(a), we can clearly see that the system has complex dynamic behaviors such as periodic orbit, chaos, and hyperchaos. When $a \in[-1,-0.03]$ and $a \in(0.22,0.3)$, the system is a periodic orbit, and Figure 4 are the phase portraits of system (2) when $a=-1$ (the LEs are $0,-0.0424,-0.4394,-0.6696$, and $-3.4835)$. When $a=0.2$, the LEs are $0.0790,-0.0078$, $-0.0627,-0.1078$, and -1.9274 , with a positive Lyapunov exponent, so system (2) is in chaotic state $(a \in[0.3,0.48)$ and $a \in(-0.03,0.22])$. When $a \in[0.48,1]$, the system is hyperchaotic, and the typical four-wing hyperchaotic attractor is shown in Figure 1 . When $a=0.78$, the LEs are $\mathrm{LE} 1=0.1712, \mathrm{LE} 2=0.0907, \mathrm{LE} 3=0.0107, \mathrm{LE} 4=0$, and LE5 $=-2.3243$, and it can be judged that system (2) is hyperchaotic and has three positive Lyapunov exponents.
Figure 3(b) describes the bifurcation diagram of system (2) varying with parameter $a$. With the increase of $a$, the system changes from period to chaos. The Kaplan-Yorke dimension of system (2) can be calculated by the following formula:

$$
D_{L}=j+\sum_{i=1}^{j} \frac{L j}{|L j+1|}=4+\frac{0.1712+0.0907+0.0107-0}{2.3243}=4.1172 \text {, }
$$

where $j$ is the largest integer satisfying $\sum_{i=1}^{j} L j \geq 0$ and $\sum_{i=1}^{j} L j<0$. It can be seen from $D_{L}=4.1172$ that the Lyapunov dimension of system (2) is fractional. Therefore, the proposed 5D FWMHS is a real hyperchaotic system with strong complexity.

As an important analytical tool, the Poincare map is used to further study the dynamic characteristics of the $5 \mathrm{D}$ HFWMS. Figure 5(a) shows the Poincare map of the $x-y$ plane at $z=0$ and four branches can be seen; Figure 5(b) shows the Poincare map of the $x-z$ plane at $y=0$ and has many branches; Figure 5(c) shows the Poincare map of the $y-z$ plane at $w=0$ and the outline of the four wings can be seen, indicating the existence of the four-wing phenomenon. Figure 5 shows that system (2) has a four-wing chaotic attractor with fractal structure.

2.4.2. Two-Wing Hyperchaotic Attractor. When the system parameters are chosen as $a=11, c=0.7, m=1, d=0.2, e=$ 0.1 , and $n=0.01$ and the initial condition is set to $[0,1,0,0,0]$, and the numerical results of the Lyapunov exponent varying with the system parameter $b$ is shown in Figure 6(a). Figure 6(b) is a bifurcation diagram corresponding to Figure 6(a). From Figure 6, we can see that system (2) has more complex dynamic behavior in the parameter $b \in[-10,0]$ interval, such as quasi-periodicity, period, chaos, and hyperchaos. Table 1 classifies these dynamical behaviors and then makes a detailed analysis of LEs and their dynamical behaviors varying with parameter $b$ as follows:

(i) When $b \in(-4.4,0]$, the Lyapunov exponents of system (2) at $b=-1$ are $0.5791,0.1087,-0.0316$, -0.2607 , and -4.5443 . The system is in a hyperchaotic state, and the corresponding phase portraits are shown in Figure $7(\mathrm{a})$.

(ii) When $b \in[-5.55,-4.4]$, the Lyapunov exponents of system (2) at $b=-5$ are $0,-0.0132,-0.2670$, -0.4978 , and -2.1754 . The system is in a period -5 state, and the corresponding phase portraits are shown in Figure 7(b).

(iii) When $b \in[-7,-6.6]$ and $b \in[-10,-7.2]$, the Lyapunov exponents of system (2) at $b=-8$ are 0.0279 , $-0.0271,-0.2093,-0.3481$, and -1.9681 . The phase portraits shown in Figure 7 (c) show that the system is in a quasi-periodic state.

(iv) When $b \in(-6.6,-5.55)$ and $b \in(-7.2,-7)$, the Lyapunov exponents of system (2) at $b=-6.15$ are $0.0918,-0.0091,-0.16-0.3278$, and -2.2332 . The 


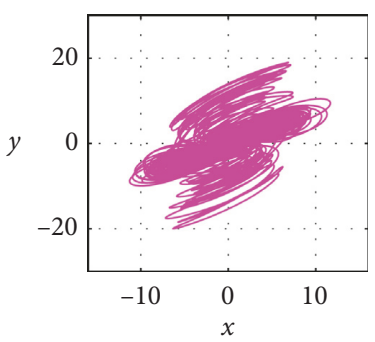

(a)

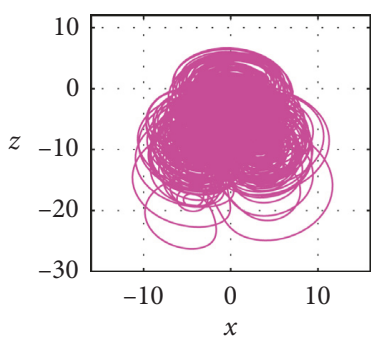

(b)

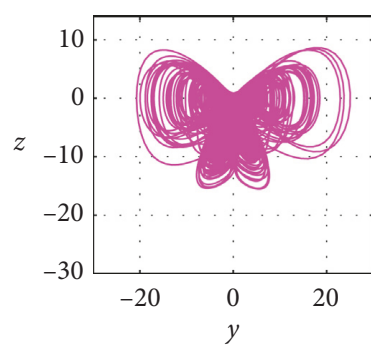

(c)

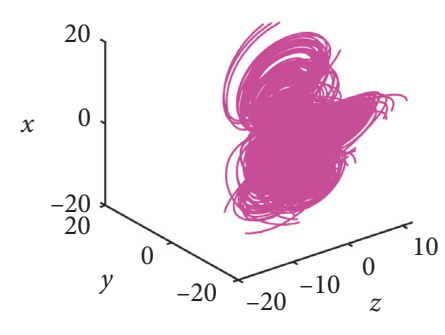

(d)

FIGURE 1: The four-wing hyperchaotic attractor of system (2): (a) in the $x-y$ plane, (b) in the $x-z$ plane, (c) in the $y-z$ plane, and (d) in the $x-y-z$ space.

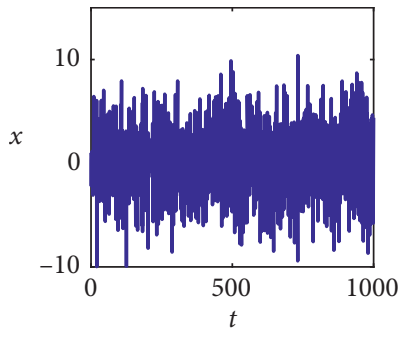

(a)

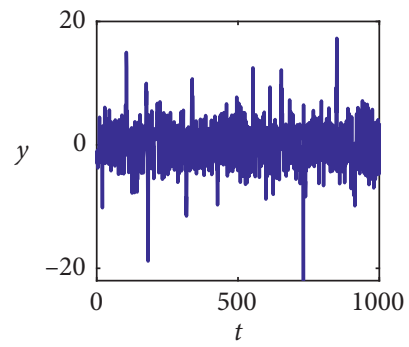

(b)

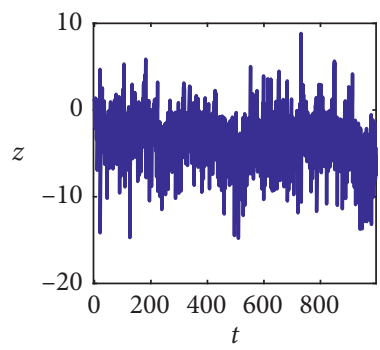

(c)

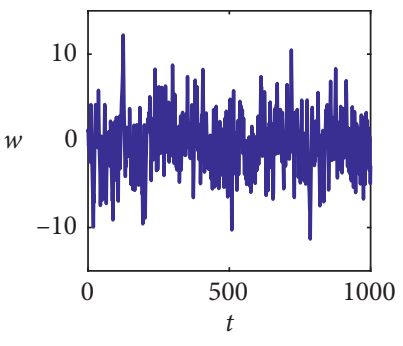

(d)

Figure 2: The time variation of state equations $x, y, z$, and $w$ of system (2): (a) $t-x$, (b) $t-y$, (c) $t-z$, and (d) $t-w$.

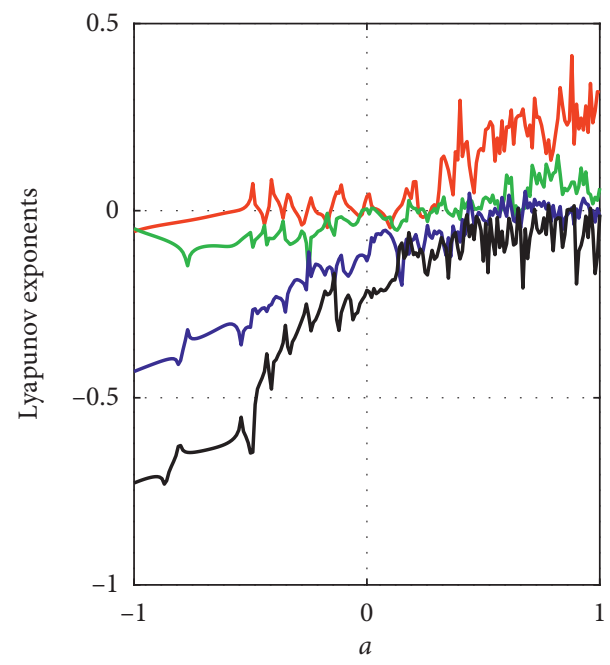

(a)

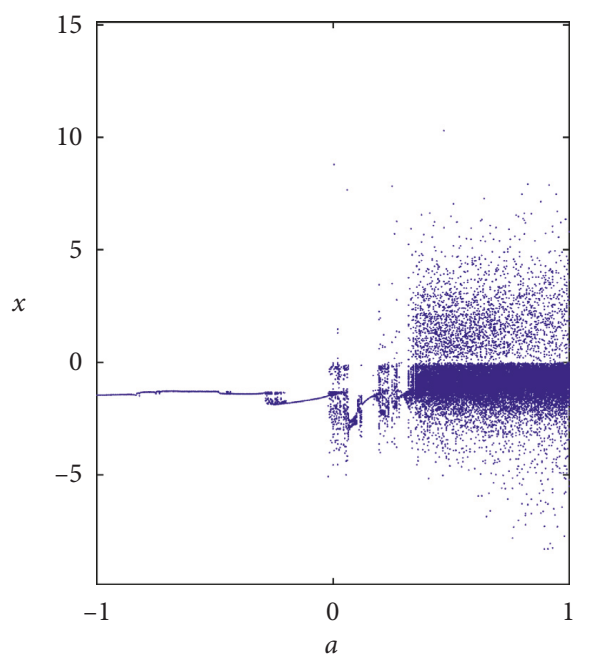

(b)

FIGURE 3: Lyapunov exponents and bifurcation diagram for increasing parameter $a$ (the five LE5 is out of plot).

system shows a chaotic state, and the corresponding phase portraits are shown in Figure 7(d).

2.4.3. Complexity Analysis by Spectral Entropy. The complexity of spectral entropy (SE) is based on the discrete Fourier transformation. The distribution of energy in Fourier transform domain is calculated, and then the SE value is calculated by Shannon entropy, which reflects the disorder of time series in frequency domain [61, 101, 102]. The chaotic diagram using the complexity of SE usually reflects the spatial complexity of chaotic system parameters. In this section, the SE algorithm is used to analyze the complexity of system (2). Figure 8 shows the complexity of SE of system (2) under initial condition $[1,-1,1,1,1]$. It can be seen that Figure 8(a) corresponds well to the maximum Lyapunov exponent in Figure 3. The control parameters $a$ and $b$ of the chaotic system are divided into $101 \times 101$ parts, 


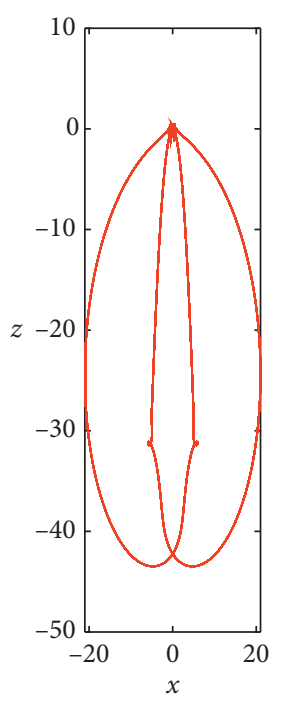

(a)

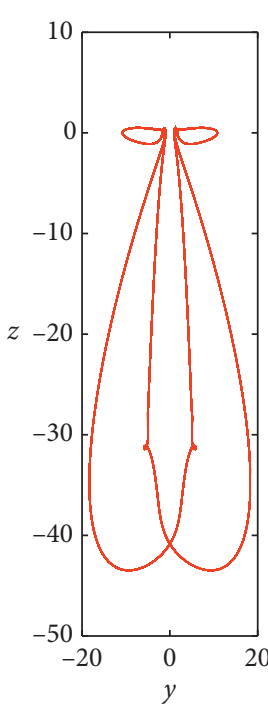

(b)

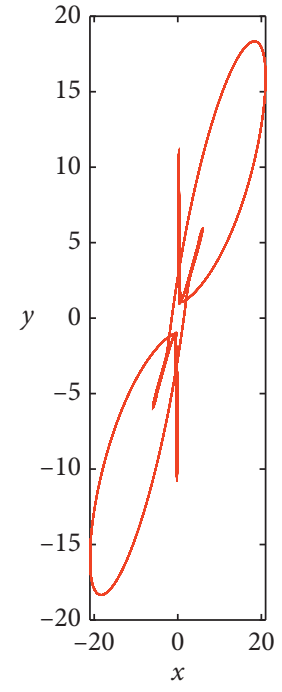

(c)

Figure 4: Periodic phase portraits of system (2) with $a=-1$ : (a) in the $x-z$ plane, (b) in the $y-z$ plane, and (c) in the $x-y$ plane.

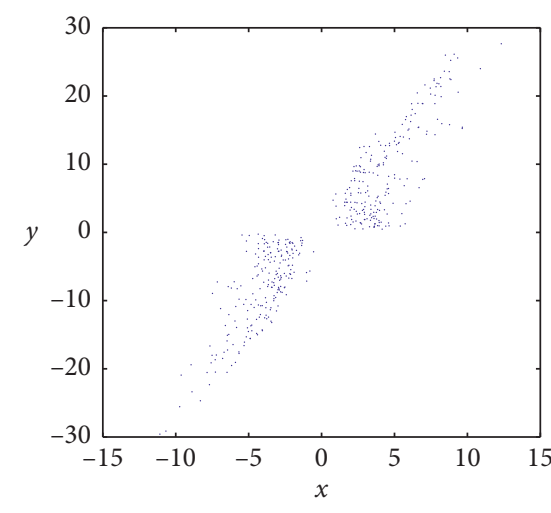

(a)

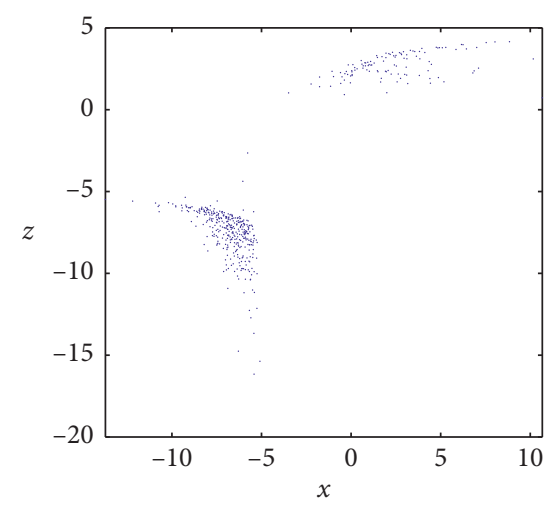

(b)

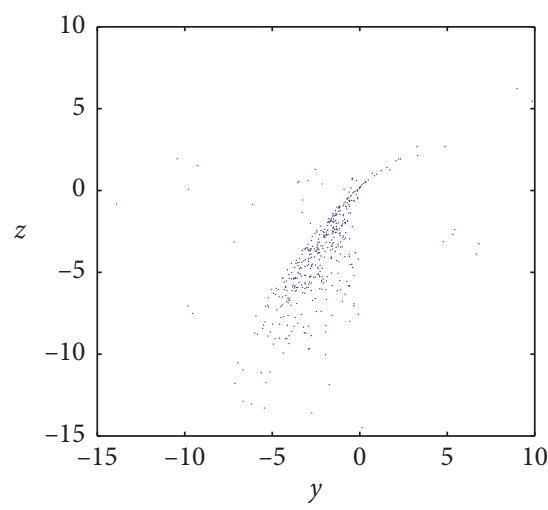

(c)

FIGURE 5: Poincare map of system (2) with parameters $a=1$ : (a) projection on $x-y$ plane with $z=0$, (b) projection on $x-z$ plane with $y=0$, and (c) projection on $y-z$ plane with $w=0$.

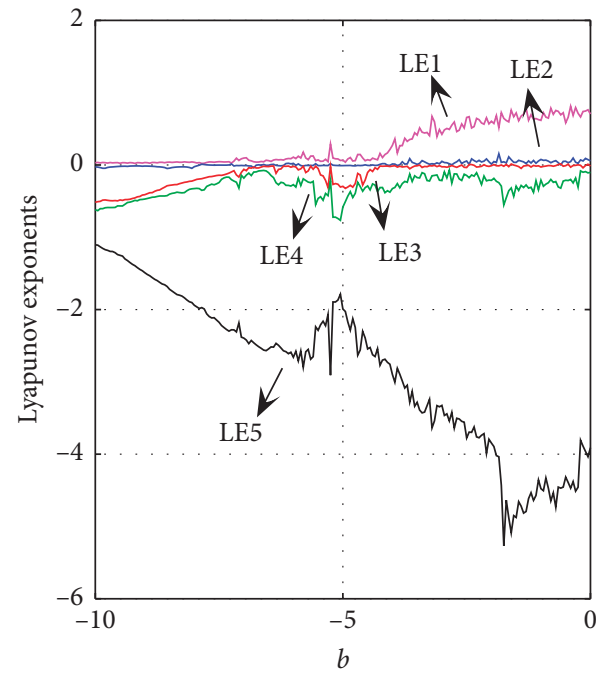

(a)

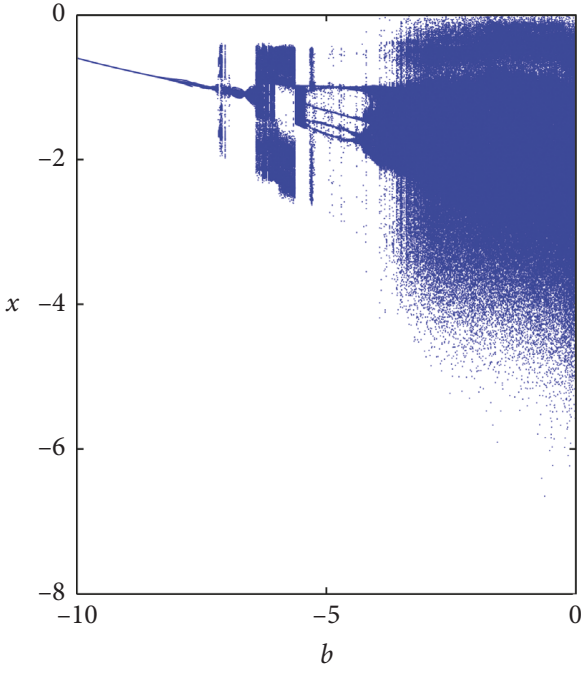

(b)

FIGURE 6: Lyapunov exponents and bifurcation diagram for increasing parameter $b$. 
TABLE 1: The LEs and dynamical behavior under different parameter range of $b$.

\begin{tabular}{lcc}
\hline$b$ & (LE1, LE2, LE3, LE4, LE5) & Dynamic \\
\hline$(-4.4,0]$ & $(+,+, 0,-,-)$ & Hyperchaotic \\
{$[-5.55,-4.4]$} & $(0,-,-,-,-)$ & Periodic \\
$(-6.6,-5.55)$ & $(+, 0,-,-,-)$ & Chaotic \\
{$[-7,-6.6]$} & $(0,0,-,-,-)$ & Torus \\
$(-7.2,-7)$ & $(+, 0,-,-,-)$ & Chaotic \\
{$[-10,-7.2]$} & $(0,0,-,-,-)$ & Quasi-periodic \\
\hline
\end{tabular}
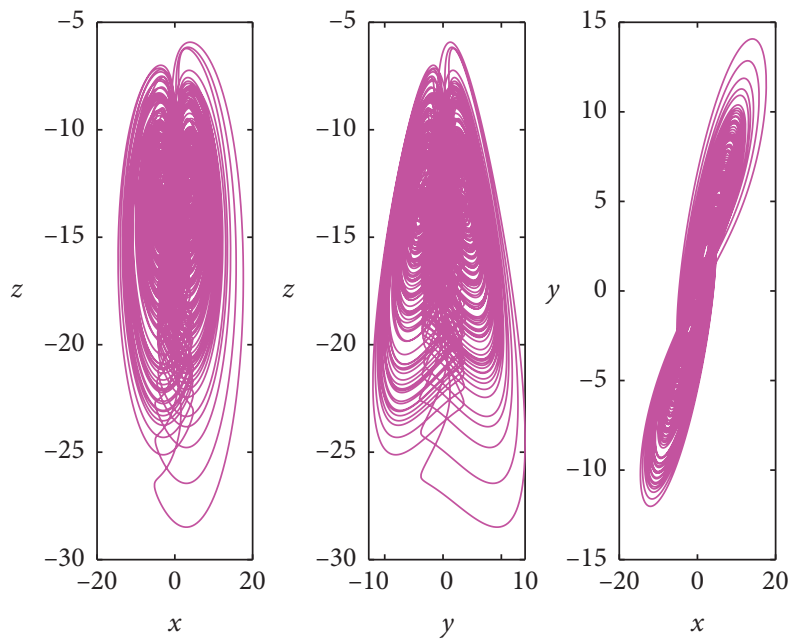

(a)
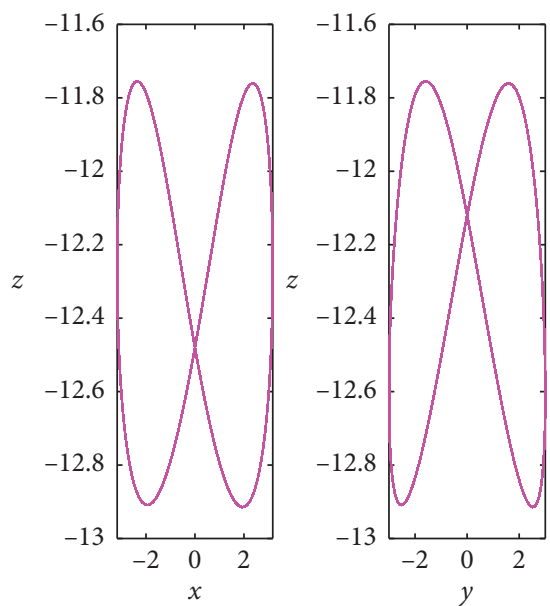

(c)

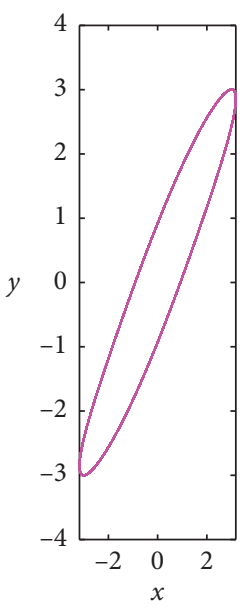

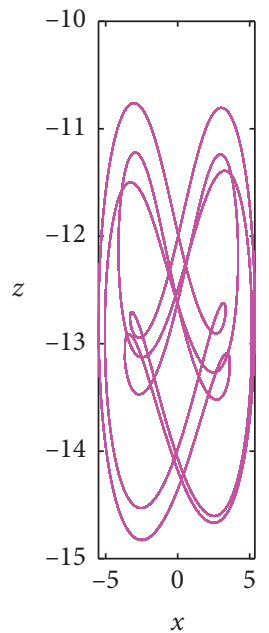
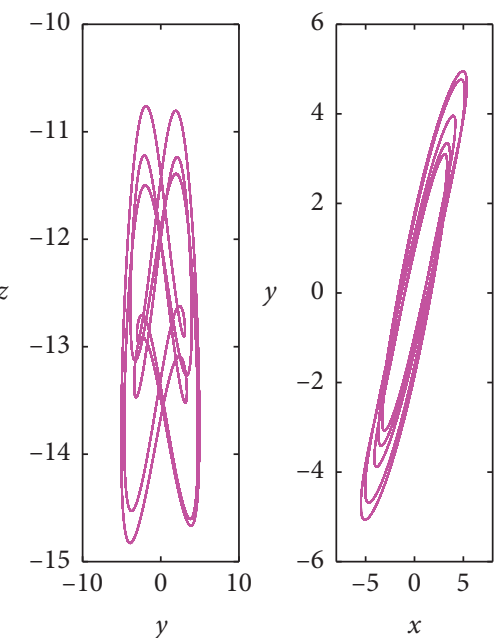

(b)
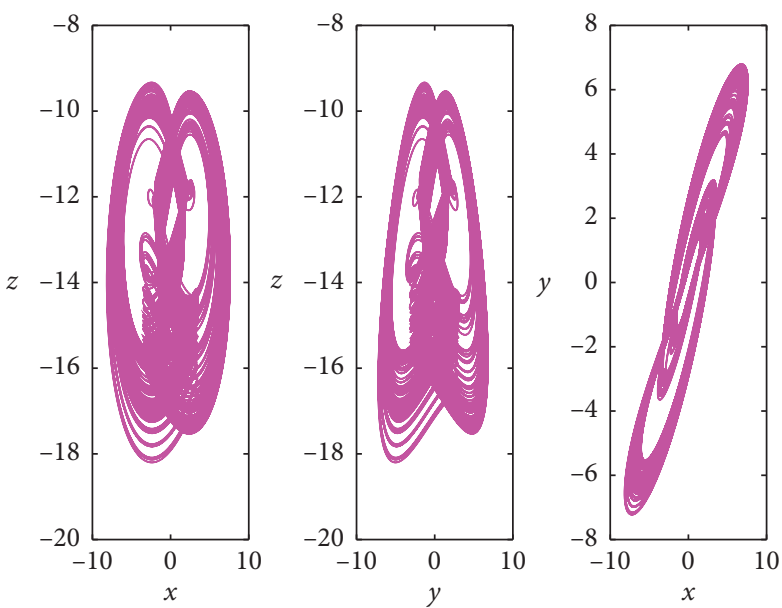

(d)

Figure 7: Simulated phase portraits of system (2) with different $b$ : (a) $b=-1$, (b) $b=-5$, (c) $b=-8$, and (d) $b=-6.15$.

including $a \in[-1,1]$ and $b \in[0,5]$. Then, calculate the SE of each point $(a, b)$ in the parameter space, as shown in Figure 8(b). The results show that with the increase of parameter $a$, the more complex the chaotic system is, and the higher the complexity of the system is mainly concentrated in $a \in[0.5,1]$. Figure 9 is the SE diagram of system (2) under initial condition $[0,1,0,0,0]$, where $a \in[10,20]$ and $b \in[-10,0]$. Figure 9(a) corresponds well to the maximum Lyapunov exponent in Figure 6. Figure 9(b) shows the complexity of SE in the plane of control parameters $a$ and $b$. The results show that when $a=11$, with the increase of parameter $b$, the larger the $\mathrm{SE}$ value is, and the higher the complexity of the system is mainly concentrated in $b \in[-3.8,0]$.

\section{The FPGA-Based Model of the Novel 5D HFWMS}

Devices in analog circuits are easy to aging and inflexible, which makes more and more researchers begin to pay attention to digital devices on the FPGA. With the characteristics of high-speed operation, high integration, and free 


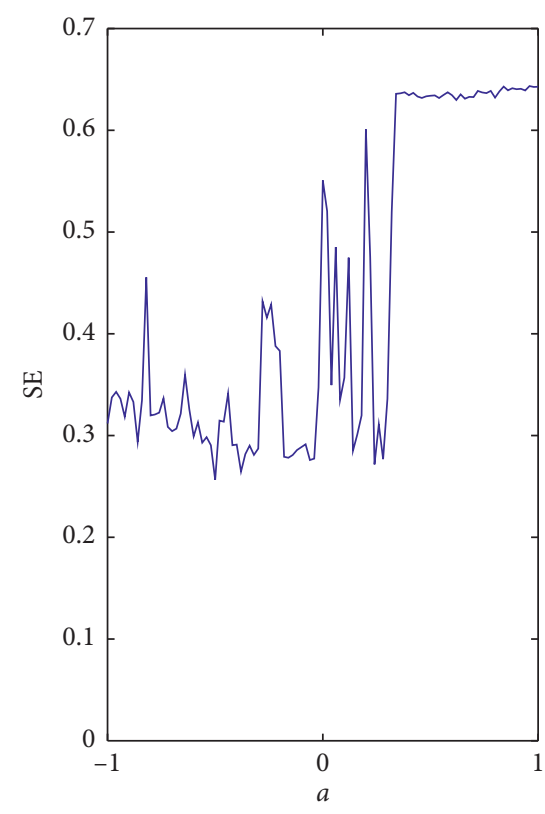

(a)

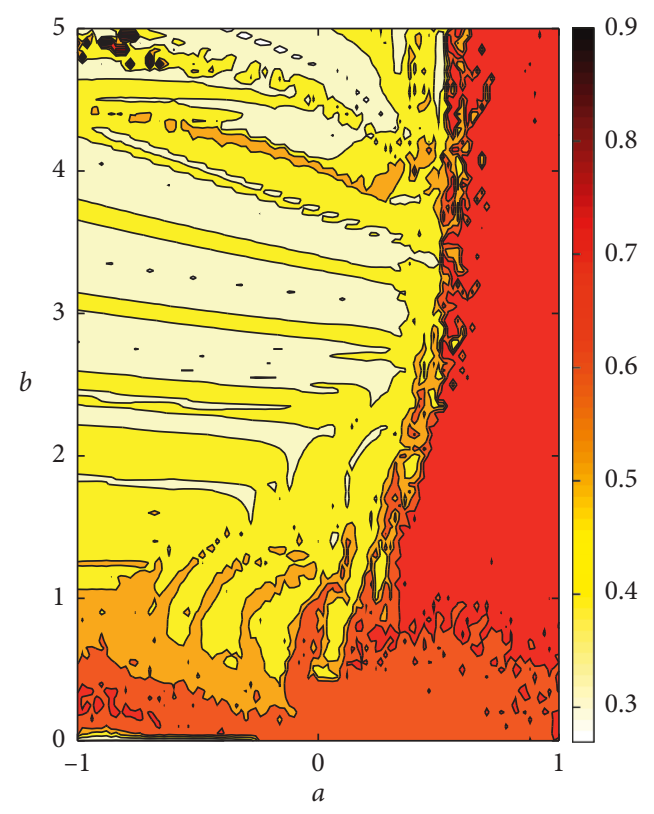

(b)

FIGURE 8: The complexity of SE of system (2): (a) SE versus $a$ when $b=1$ and (b) in the $a-b$ plane.

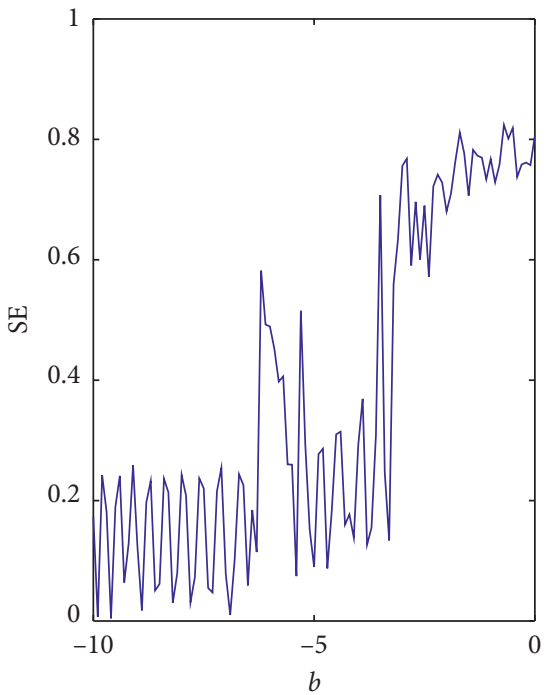

(a)

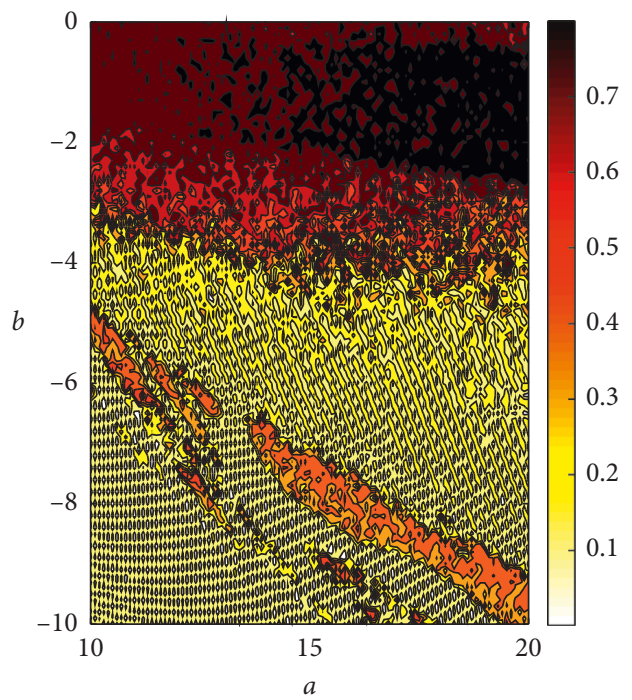

(b)

FIgURE 9: The complexity of SE of system (2): (a) SE versus $b$ when $a=11$ and (b) in the $a-b$ plane.

design, FPGA can easily generate chaotic signals. Nowadays, many numerical algorithms are used to solve the nonlinear differential equations of chaotic systems. The Euler algorithm is the simplest of all algorithms, but its accuracy is not high $[64,65]$. The Heun algorithm is more sensitive than the Euler method [63]. The Runge-Kutta algorithm is better than other algorithms in operation effect, with high accuracy, stable calculation process, and easy realization. RK-4 is easier to implement than RK-5, so RK-4 is widely used to solve chaotic systems [103]. Equation (12) gives the formula for calculating $K_{1}, K_{2}, K_{3}$, and $K_{4}$, which represents the slope value of $\left[y_{0}, y_{i}\right]$ :

$$
\begin{aligned}
& t_{i+1}=t_{i}+h, \\
& K_{1}=f\left(t_{i}, y_{i}\right), \\
& K_{2}=f\left(t_{i}+\frac{h}{2}, y_{i}+\frac{h}{2} K_{1}\right), \\
& K_{3}=f\left(t_{i}+\frac{h}{2}, y_{i}+\frac{h}{2} K_{2}\right), \\
& K_{4}=f\left(t_{i}+h, y_{i}+h K_{3}\right) .
\end{aligned}
$$


For the designed HFWMS, the initial conditions $x(0)=$ $1, y(0)=-1, z(0)=1, w(0)=1$, and $\varphi(0)=1$ and the step size $h=0.01$ are given. Five equations in HFWMS are calculated according to the RK-4 algorithm flow chart and equation (12). By using the RK-4 algorithm of Verilog, the designed HFWMS is implemented on FPGA. The central idea of designing a chaotic signal generator with FPGA is to divide the whole system into several functional modules, including RK-4 solving module, data selector module, ys module, and numerical conversion module. The function module contains many arithmetic units, including multiplier, adder, and subtractor. These arithmetic units are created in cooperation with the IP core generator and follow the standard of IEEE 754.

The top-level block diagram of the chaotic signal generator based on FPGA using the RK-4 algorithm is shown in Figure 10. As can be seen from Figure 10, the design system has three inputs and six outputs. The output signal consists of five 32-bit output signals ( $X_{-}$out, $Y_{-}$out, $Z_{-}$out, $W_{\text {_out }}$, and $\varphi_{-}$out) and 1 bit flag signal $X Y Z W \varphi_{-}$ready. When the calculation produces $X_{-}$out, $Y_{-}$out, $Z_{-}$out, $W_{-}$out, and $\varphi_{-}$out, the signal $X Y Z W \varphi_{-}$ready will be set to a valid bit. Clock signal (Clk) and Reset are both 1 bit signals, which are used to ensure synchronization between the system and other modules; 32 bit $\Delta h$ represents step size, which is used to determine the sensitivity of the algorithm.

The second block diagram of the chaotic signal generator based on FPGA using the RK-4 algorithm is displayed in Figure 11, which consists of a Multipler (MUX) and 5D HFWMS oscillator. As can be seen from that the MUX unit is used to obtain the initial condition signal at the first operation. These signals were initially defined by the designer, and then obtained as feedback signals by the output signals ( $X_{-}$out, $Y_{-}$out, $Z_{-}$out, $W_{-}$out, and $\varphi_{-}$out). Figure 12 is the third block diagram of the chaotic signal generator based on FPGA using the RK-4 algorithm, which consists of three parts: MUX unit, HFWMS oscillator unit, and data processing unit. $K 1$ unit, $K 2$ unit, $K 3$ unit, $K 4$ unit, and $y s$ are important components of the HFWMS oscillator, which are pipelined structures used in the calculation of RungeKutta algorithm. ys unit can make the chaotic oscillator produce output signal in a definite clock period. I.C. (initial conditions) are initially defined by the designer. When HFWMS generates a set of output values, the value of $X Y Z W \varphi_{-}$ready is set to a valid bit and the values of $x(k+1), y(k+1), z(k+1), w(k+1)$, and $\varphi(k+1)$ generated by the oscillator are fed back to MUX as the initial values of the next operation. The data processing unit has two functions: (1) converting 32-bit floating-point signals ( $X_{-}$out, $Y_{-}$out, $Z_{-}$out, $W_{\text {_out, }}$ and $\varphi_{\text {_out }}$ generated by the oscillator into 14-bit fixed-point signals and (2) converting signed fixed-point signals into unsigned fixed-point signals. The digital-to-analog converter AN9767 (DAC) converts 14digit digital signals into analog signals for easy display on the oscilloscope.

The digital hardware implementation of the $5 \mathrm{D}$ hyperchaotic oscillator based on RK-4 has been synthesized on the Xilinx ZYNQ-XC7Z020 FPGA chip. This design is implemented, synthesized, and downloaded using Vivado 2018.3.

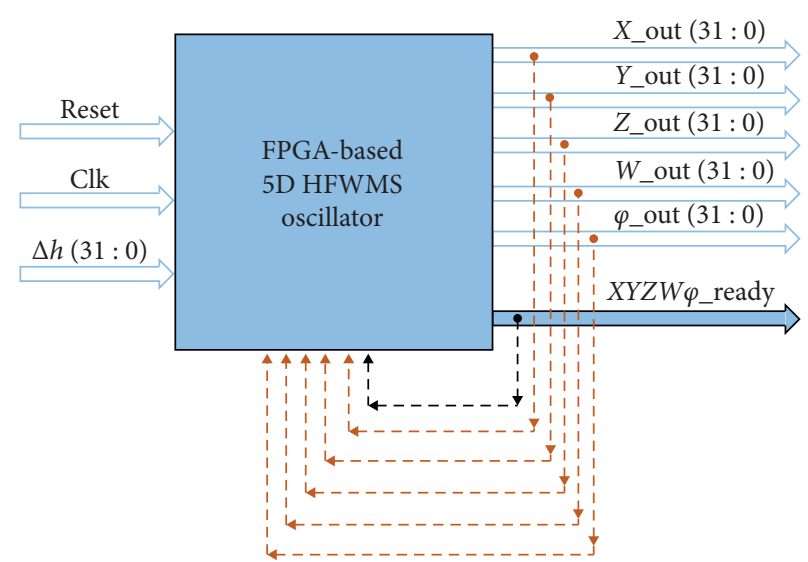

Figure 10: The top-level block diagram of the FPGA-based 5D HFWMS.

The parameter statistics of the related resource utilization of the FPGA and the clock speed of each module are calculated. In order to better analyze the experimental results, we convert the experimental data into hexadecimal. Figure 13 shows a discrete time series ( $X_{-}$out, $Y_{-}$out, $Z_{-}$out, $W_{-}$out, and $\varphi_{-}$out) obtained for the HFWMS oscillator based on FPGA, which corresponds to the $x, y, z, w$, and $\varphi$ signals of the continuous chaotic system. Figure 14 are the phase portraits of $Y_{-}$out and $Z_{-}$out which are displayed by the $5 \mathrm{D}$ HFWMS on the oscilloscope. From Figure 14, it can be seen that several kinds of phase portraits designed based on FPGA are consistent with the Matlab simulation diagrams, which means that the designed HFWMS based on FPGA can be implemented well. Table 2 provides statistical data on resource utilization, chip speed, and performance of the Xilinx ZYNQ-XC7Z020 FPGA chip. The minimum clock period and maximum operating frequency of HFWMS based on FPGA are $6.763 \mathrm{~ns}$ and $147.863 \mathrm{MHz}$.

\subsection{Active Control Synchronization and Secure Communication of the Novel 5D HFWMS}

3.1.1. Synchronization of the Novel 5D HFWMS by the Active Control Method. Synchronization design is the key to secure communication. Therefore, it is necessary to synchronize the designed chaotic system before realizing secure communication. A synchronization system consists of two parts, one is the master system and the other is the slave system. In this section, the active control method is used to synchronize system (2), and system (2) is set as the master system and rewritten as

$$
\left\{\begin{array}{l}
\dot{x}_{1}=a x_{1}+d W\left(\varphi_{1}\right) y_{1}+y_{1} z_{1}-c w_{1}, \\
\dot{y}_{1}=y_{1} z_{1}-x_{1} z_{1} \\
\dot{z}_{1}=-z_{1}-m x_{1} y_{1}+b \\
\dot{w}_{1}=x_{1} \\
\dot{\varphi}_{1}=y_{1} .
\end{array}\right.
$$

Here, the slave system uses a 5D hyperchaotic system proposed by Yang and Bai [104], which is described as 


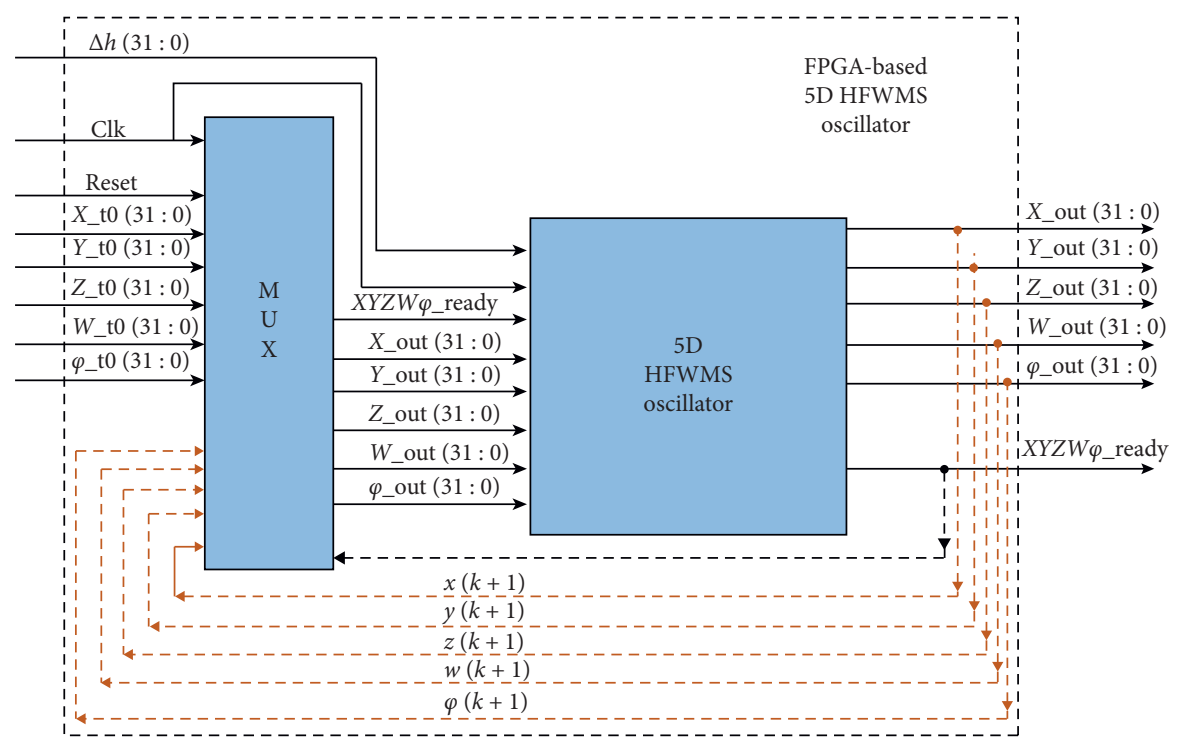

Figure 11: The second-level block diagram of the FPGA-based 5D HFWMS.

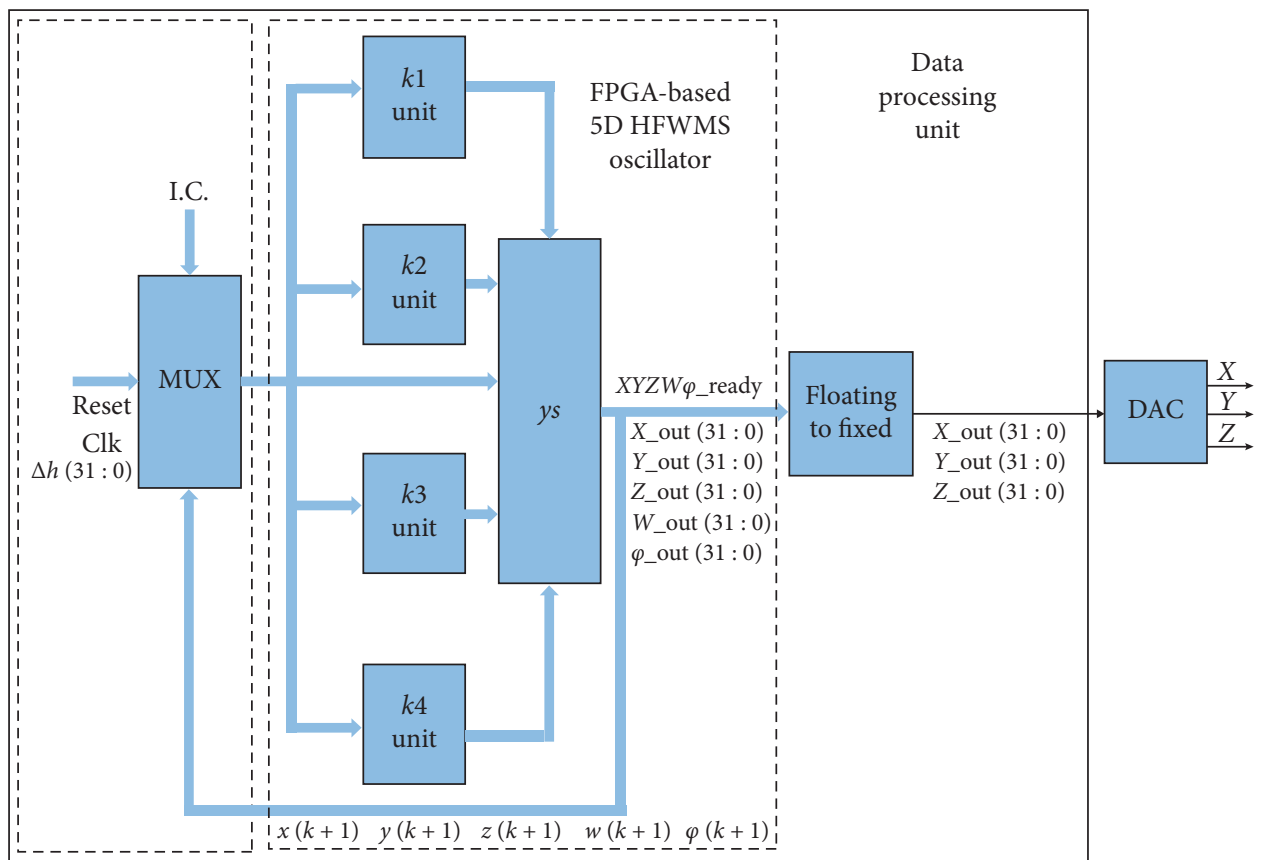

Figure 12: The third-level block diagram of the FPGA-based 5D HFWMS.

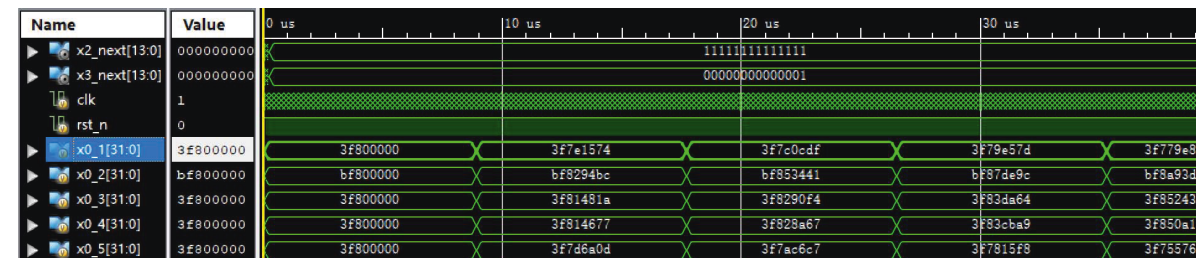

FIGURE 13: Simulation results of the FPGA-based HFWMS signal generator with the RK-4 algorithm. 


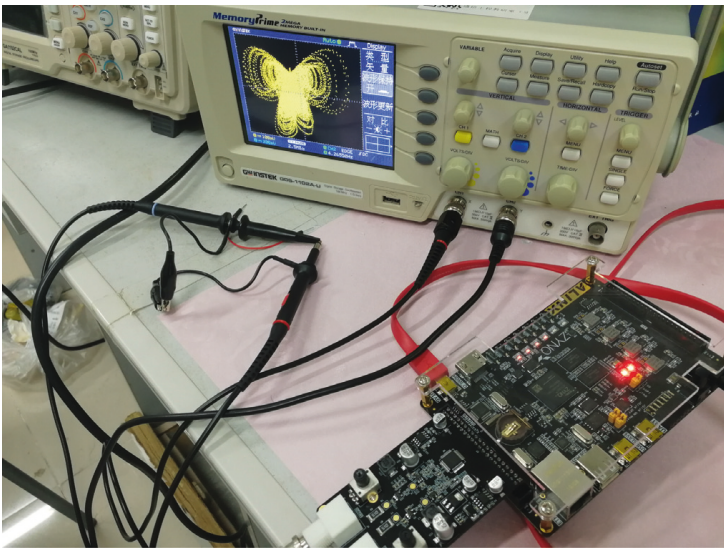

(a)

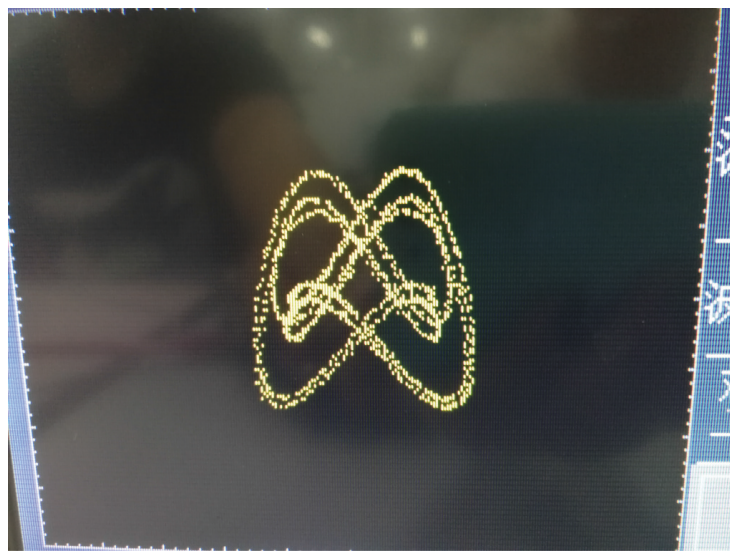

(c)

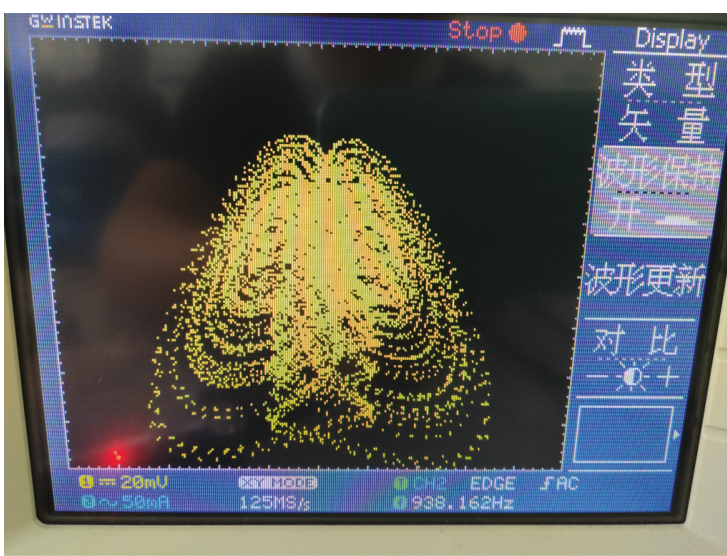

(b)

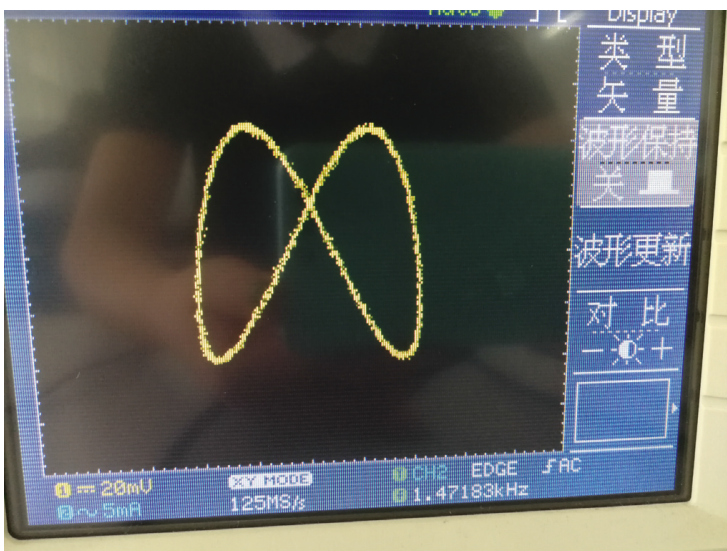

(d)

FIGURE 14: Implementation platform and exemplificative phase portraits generated by the FPGA implementation of the proposed HFWMS: (a) four-wing hyperchaotic attractor, (b) two-wing hyperchaotic attractor, (c) period-5 state, and (d) quasi-periodic state.

TABLE 2: Xilinx ZYNQ-XC7Z020 FPGA chip hardware usage statistics of the 5D HFWMS.

\begin{tabular}{lccc}
\hline Resource & Used & Available & Utilization (\%) \\
\hline Slice register & 26893 & 106400 & 53200 \\
Number of slice LUTs & 23173 & 125 & 43 \\
Number of bonded IOBs & 30 & 32 & 24 \\
Number of BUFG & 1 & - & 3 \\
Max. clock frequency & $147.863 \mathrm{MHz}$ & - & - \\
Latency & $13.53 \mathrm{~ns}$ & - & - \\
Throughput & $59.15 \mathrm{Mbit} / \mathrm{s}$ & - & - \\
Power & $0.275 \mathrm{~W}$ & & - \\
\hline
\end{tabular}

$$
\left\{\begin{array}{l}
\dot{x}_{2}=a_{1}\left(y_{2}-x_{2}\right)+u_{1}, \\
\dot{y}_{2}=c_{1} x_{2}+d_{1} y_{2}-x_{2} z_{2}+\varphi_{2}+u_{2}, \\
\dot{z}_{2}=-b_{1} z_{2}-x_{2}^{2}+u_{3}, \\
\dot{w}_{2}=g_{1} y_{2}+f_{1} w_{2}+u_{4}, \\
\dot{\varphi}_{2}=-r_{1} x_{2}-k_{1} \varphi_{2}+u_{5},
\end{array}\right.
$$

where $a_{1}, b_{1}, c_{1}, d_{1}, g_{1}, f_{1}, r_{1}$, and $k_{1}$ are the system parameters and $u=\left[u_{1}, u_{2}, u_{3}, u_{4}, u_{5}\right]^{T}$ is the active controller of synchronous systems, which can make the master and slave systems gradually synchronize under different initial conditions. Define the errors as

$$
\left\{\begin{array}{l}
e_{1}=x_{2}-x_{1} \\
e_{2}=y_{2}-y_{1} \\
e_{3}=z_{2}-z_{1} \\
e_{4}=w_{2}-w_{1} \\
e_{5}=\varphi_{2}-\varphi_{1}
\end{array}\right.
$$

Then, the error dynamic system can be obtained as 


$$
\left\{\begin{array}{l}
\dot{e}_{1}=\left(a-a_{1}\right) e_{1}+\left(a_{1}+d g\right) e_{2}-c e_{4}+c w_{2}-a x_{2}-d g y_{2}+a_{1}\left(y_{1}-x_{1}\right)-y_{1} z_{1}-3 d n \varphi_{1}^{2} y_{1}+u_{1}, \\
\dot{e}_{2}=c_{1} e_{1}+d_{1} e_{2}+e_{5}+\varphi_{1}-x_{2} z_{2}+x_{1} z_{1}-y_{1} z_{1}+c_{1} x_{1}+d_{1} y_{1}+u_{2}, \\
\dot{e}_{3}=-b_{1} e_{3}-e_{3}+x_{2}^{2}+m x_{1} y_{1}-b+z_{2}-b_{1} z_{1}+u_{3}, \\
\dot{e}_{4}=g_{1} e_{1}+g_{1} y_{1}+f_{1} e_{4}+f_{1} w_{1}+e_{1}-x_{2}+u_{4}, \\
\dot{e}_{5}=-r_{1} e_{1}-k_{1} e_{5}-r_{1} x_{1}-k_{1} \varphi_{1}-y_{2}+e_{2}+u_{5} .
\end{array}\right.
$$

By simplifying the linear term of equation (16), the active controller can be obtained as

$$
\left\{\begin{array}{l}
u_{1}=-c w_{2}+a x_{2}+d g y_{2}-a_{1}\left(y_{1}-x_{1}\right)+y_{1} z_{1}+3 d n \varphi_{1}^{2} y_{1}+v_{1} \\
u_{2}=-\varphi_{1}+x_{2} z_{2}-x_{1} z_{1}+y_{1} z_{1}-c_{1} x_{1}-d_{1} y_{1}+v_{2} \\
u_{3}=-x_{2}^{2}-m x_{1} y_{1}+b-z_{2}+b_{1} z_{1}+v_{3} \\
u_{4}=-g_{1} y_{1}-f_{1} w_{1}+x_{2}+v_{4} \\
u_{5}=r_{1} x_{1}+k_{1} \varphi_{1}+y_{2}+v_{5}
\end{array}\right.
$$

where $v=\left[v_{1}, v_{2}, v_{3}, v_{4}, v_{5}\right]^{T}$ is the control input. The substitution of (17) in (16) leads to a linear error dynamics equation without the active controller:

$$
\left\{\begin{array}{l}
\dot{e}_{1}=\left(a-a_{1}\right) e_{1}+\left(a_{1}+d g\right) e_{2}-c e_{4}+v_{1} \\
\dot{e}_{2}=c_{1} e_{1}+d_{1} e_{2}+e_{5}+v_{2} \\
\dot{e}_{3}=-b_{1} e_{3}-e_{3}+v_{3} \\
\dot{e}_{4}=g_{1} e_{1}+f_{1} e_{4}+e_{1}+v_{4} \\
\dot{e}_{5}=-r_{1} e_{1}-k_{1} e_{5}+e_{2}+v_{5}
\end{array}\right.
$$

In order to achieve synchronization of different structures, it is necessary to

$$
\lim _{x \longrightarrow \infty} e_{i}=0, \quad i=1,2,3,4,5 .
$$

Equation (17) shows that if system (18) tends to be stable over time and under the control of input $v=\left[v_{1}\right.$, $\left.v_{2}, v_{3}, v_{4}, v_{5}\right]^{T}$, the error variable $e=\left[e_{1}, e_{2}, e_{3}, e_{4}, e_{5}\right]^{T}$ tends to zero, and then the master system (12) and the slave system (13) realize the synchronization with different structures. To achieve this goal, we define a matrix $A$ to represent the relationship between the error system and the control input, which can be expressed as

$$
v=A \cdot e .
$$

According to the stability criterion, if equation (20) is stable, all eigenvalues of matrix A are negative. Thus, equation (20) can be expressed as

$$
\left[\begin{array}{c}
v_{1} \\
v_{2} \\
v_{3} \\
v_{4} \\
v_{5}
\end{array}\right]=\left[\begin{array}{ccccc}
a_{1}-a-1 & -a_{1}-d g & 0 & c & 0 \\
-c_{1} & -d_{1}-1 & 0 & 0 & -1 \\
0 & 0 & b_{1} & 0 & 0 \\
-1 & -e_{1} & 0 & -f_{1}-1 & 0 \\
r_{1} & -1 & 0 & 0 & k_{1}-1
\end{array}\right]\left[\begin{array}{l}
e_{1} \\
e_{2} \\
e_{3} \\
e_{4} \\
e_{5}
\end{array}\right],
$$

where matrix $A$ is

$$
\left[\begin{array}{ccccc}
a_{1}-a-1 & -a_{1}-d g & 0 & c & 0 \\
-c_{1} & -d_{1}-1 & 0 & 0 & -1 \\
0 & 0 & b 1 & 0 & 0 \\
-1 & -e_{1} & 0 & -f_{1}-1 & 0 \\
r_{1} & -1 & 0 & 0 & k_{1}-1
\end{array}\right]
$$

Therefore, equation (21) can be transformed into

$$
\left\{\begin{array}{l}
v_{1}=\left(a_{1}-a-1\right)\left(x_{2}-x_{1}\right)+c\left(w_{2}-w_{1}\right)-\left(a_{1}+d g\right)\left(y_{2}-y_{1}\right), \\
v_{2}=-c_{1}\left(x_{2}-x_{1}\right)-\left(d_{1}+1\right)\left(y_{2}-y_{1}\right)-\left(u_{2}-u_{1}\right) \\
v_{3}=b_{1}\left(z_{2}-z_{1}\right) \\
v_{4}=-\left(x_{2}-x_{1}\right)-g_{1}\left(y_{2}-y_{1}\right)-\left(f_{1}+1\right)\left(w_{2}-w_{1}\right) \\
v_{5}=-\left(y_{2}-y_{1}\right)-k_{1}\left(\varphi_{2}-\varphi_{1}\right)+r_{1}\left(x_{2}-x_{1}\right) .
\end{array}\right.
$$

By substituting equations (23) and (17) into equation (14), the expression of slave system is obtained as follows:

$$
\left\{\begin{array}{l}
\dot{x}_{2}=-x_{2}+2 x_{1}+d\left(g+3 n \varphi_{1}^{2}\right) y_{1}+y_{1} z_{1}-c w_{1}, \\
\dot{y}_{2}=y_{1} z_{1}-x_{1} z_{1}-y_{2}+y_{1} \\
\dot{z}_{2}=-z_{2}-m x_{1} y_{1}+b \\
\dot{w}_{2}=x_{1}-w_{2}+w_{1} \\
\dot{\varphi}_{2}=y_{1}-\varphi_{2}+\varphi_{1} .
\end{array}\right.
$$

(1) Numerical Simulation Results Based on Matlab. The effectiveness of synchronization results of active control for different structures is verified by numerical simulation. Choose the parameters of system (13) as $a=1, b=1, c=0.7, m=1$, $d=0.2, g=0.1$, and $n=0.01$ and initial states as $[1,-1,1$, $1,1]$; the parameters of system (14) as $a_{1}=35, b_{1}=7, c_{1}=$ $35, d_{1}=-5, g_{1}=10.6, f_{1}=1, r_{1}=5$, and $k_{1}=0.05$ and initial states as $[0.01,-0.01,0.01,0.01,0.01]$. Figure 15 shows the 

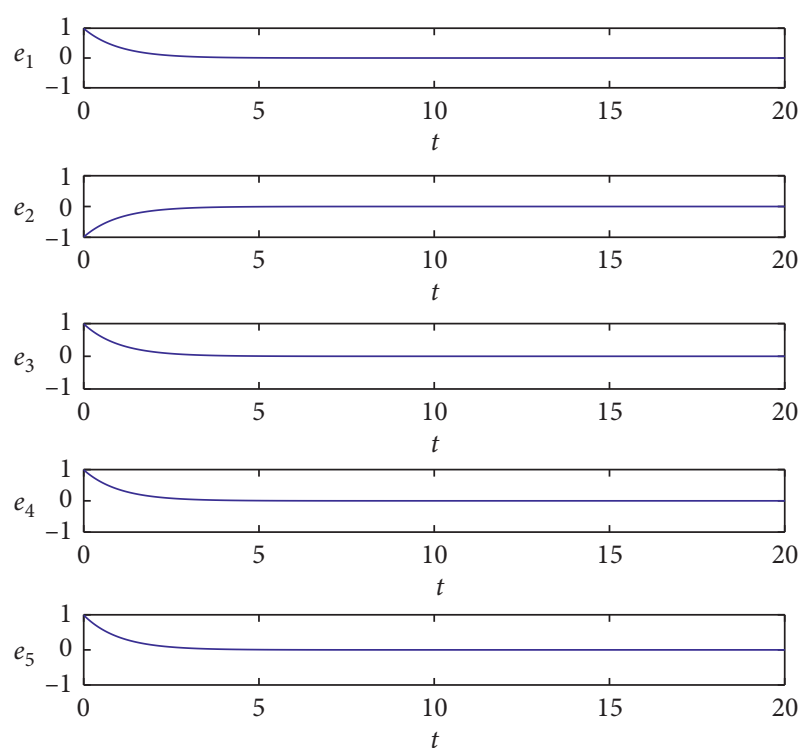

FIGURE 15: The trajectories of the synchronization errors $e_{1}, e_{2}, e_{3}, e_{4}$, and $e_{5}$ based on Matlab.

time behaviors of the error states. As we can see, the error states converge to 0 in 3 seconds, which means that the two hyperchaotic systems with different structures can achieve synchronization.

(2) Implementation Results Based on FPGA. Because of the aging and temperature change of analog devices, the device values are easy to change. Synchronization requires that the parameters of the transmitter and the receiver be highly matched. This indicates that there are a series of problems in synchronization using analog devices. It is an ideal choice to synchronize the chaotic system with FPGA, which has high reliability because it does not have the problem of temperature drift of components. In this section, according to the principle of active control synchronization, two different 5D hyperchaotic systems with different structures are synchronized on ModelSim. The Verilog design is simulated by Vivado's built-in simulator. The simulation results of synchronization errors in FPGA are shown in Figure 16. From the graph, it can be seen that all synchronization errors tend to zero. This is the same as the Matlab simulation in Figure 15 of synchronization errors. Therefore, the synchronization of $5 \mathrm{D}$ hyperchaotic systems with different structures designed by FPGA can work normally on hardware.

\subsubsection{Chaotic Masking Communication of the Novel 5D} HFWMS. The main idea of chaotic masking secure communication design is to superimpose the signal generated by the chaotic system on the useful signal to form a modulation signal, and then use the channel for transmission. The receiver demodulates the modulated signal by the output signal of the chaotic system

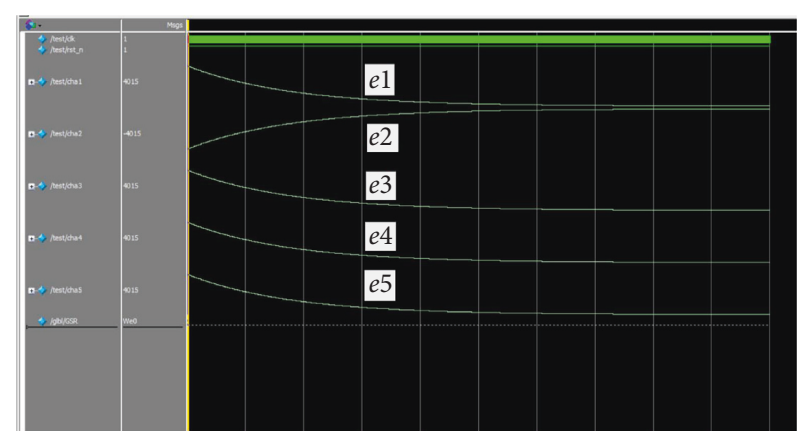

FIGURE 16: The trajectories of the synchronization errors $e_{1}, e_{2}, e_{3}, e_{4}$, and $e_{5}$ based on FPGA.

synchronized with the transmitter and recovers the original useful signal. The application of secure communication studied in this paper is based on active control synchronization between two 5D hyperchaotic systems with different structures. The schematic diagram of the designed secure communication scheme is shown in Figure 17, where $s(t)$ is the information signal, $m(t)$ is the chaotic signal generated by the master system, $p(t)$ is the mixed signal encrypted by $m(t)$ to $s(t), n(t)$ is the chaotic signal generated by the slave system, and $d(t)$ is the decrypted signal. The signal transmitted in the channel is a kind of chaotic signal similar to noise, from which it is difficult to obtain useful signals. The controller unit can synchronize the master system and slave system, which is the key of chaotic secure communication. The original useful signal can be recovered effectively by using the signal generated by the synchronized chaotic system of the controller to decrypt the encrypted signal. Set the information signal to be encrypted at the transmitter as

$$
s(t)=0.125 \sin (0.1 \pi t) .
$$

The encryption function and decryption function of the chaotic masking communication are, respectively, adopted as

$$
\begin{aligned}
& p(t)=s(t) \cdot[1+k m(t)], \\
& d(t)=\frac{p(t)}{[1+k n(t)]}
\end{aligned}
$$

The error signal between the information signal and the decrypted signal is

$$
e(t)=s(t)-d(t)
$$

(1) Numerical Simulation Results Based on Matlab. Through the encryption function, decryption function, and synchronization-related parameters, the numerical simulation results are shown in Figure 18 with $k=10$. As can be seen that the signal $p(t)$ is really difficult to be decrypted; the signal $d(t)$ generated by the decryption function corresponds well with the information signal, which 


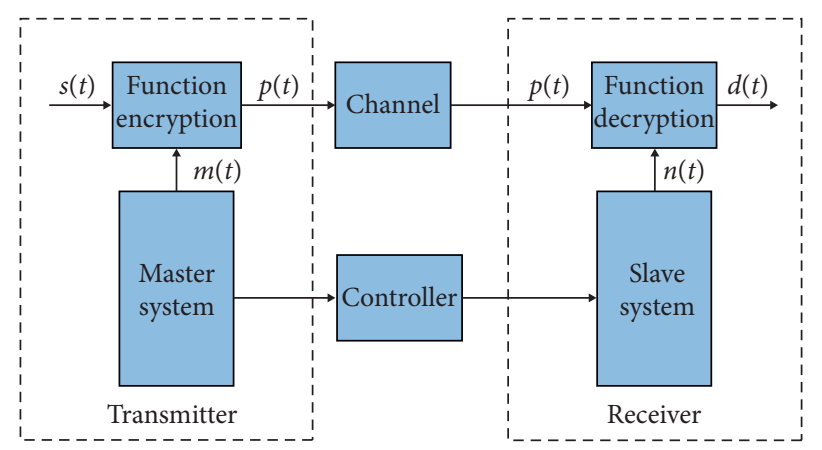

Figure 17: The schematic diagram of chaotic masking communication.

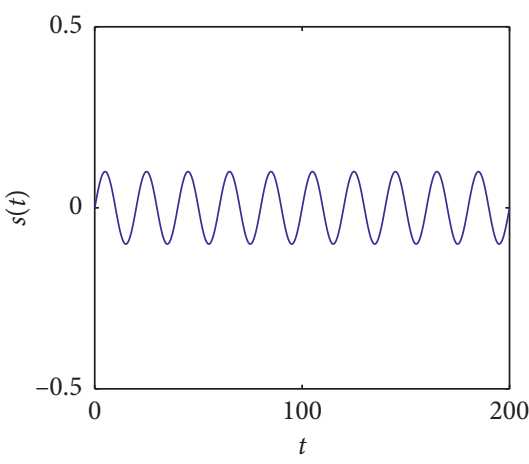

(a)

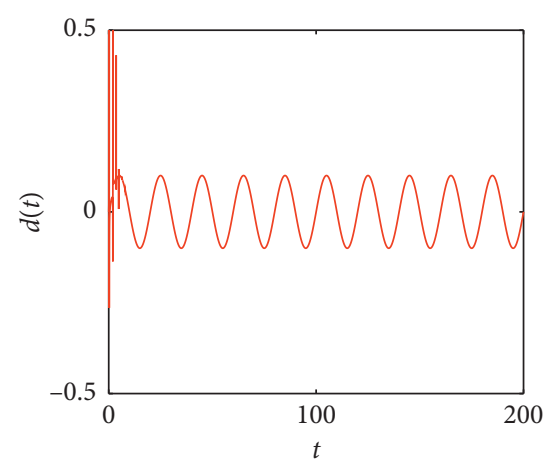

(c)

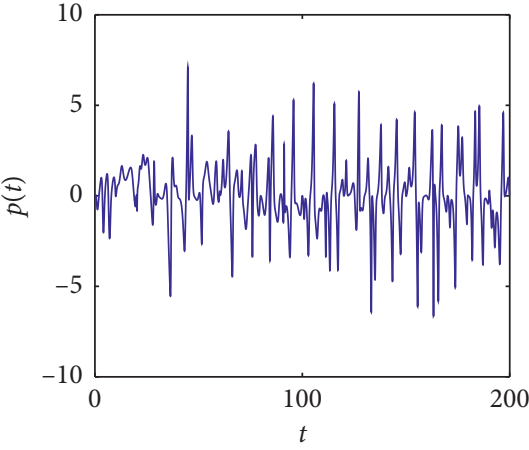

(b)

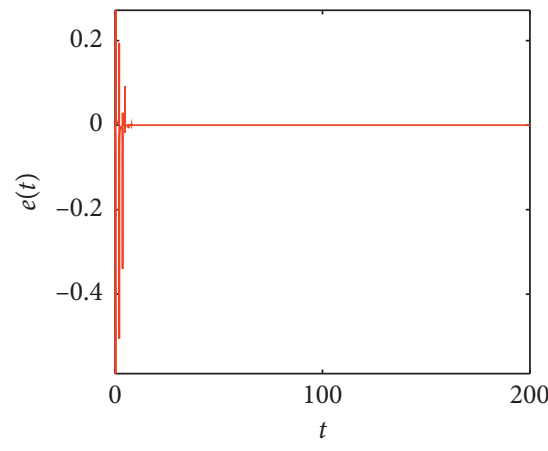

(d)

FIGURE 18: (a) Original signal $s(t)$, (b) transmitted signal $p(t)$, (c) recovered signal $d(t)$, and (d) error $e(t)=s(t)-d(t)$.

means that the receiver can recover the information signal very well.

(2) Implementation Results Based on FPGA. In order to verify the correctness of the proposed chaotic masking secure communication scheme, ModelSim is selected as the experimental platform of FGPA. According to the scheme shown in Figure 17, the hardware experiment results of the FPGA are shown in Figure 19. From Figure 19, we can see that the effective information signal $s(t)$ can be completely hidden in the chaotic sequence, the encrypted signal has strong concealment, and the received decrypted signal has good restoration quality, which is the same as the preencrypted signal.

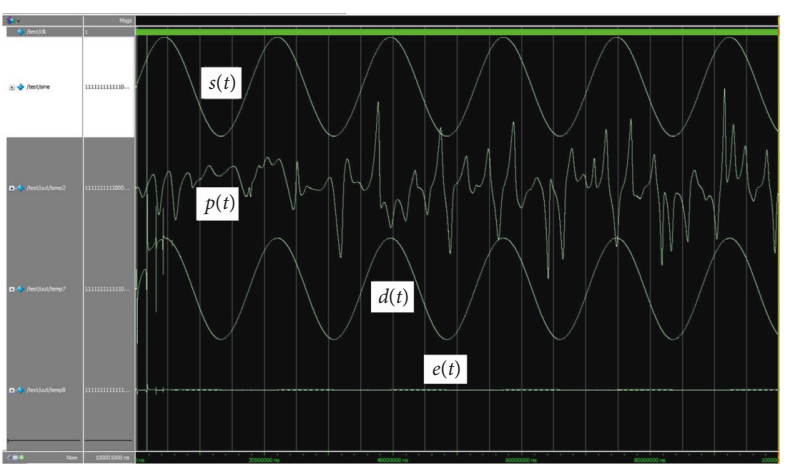

FIGURE 19: Experimental results of chaotic masking secure communication based on FPGA. 


\section{Conclusion}

In this study, a novel 5D continuous time HFWMS with multiline equilibrium and three positive Lyapunov exponents are first introduced. Dynamical analysis is performed in terms of equilibrium points, phase portraits, Poincare map, Lyapunov exponential spectrum, bifurcation diagram, and spectral entropy. Then, the four-wing, two-wing, period-5, and quasi-periodic phase portraits of the novel 5D hyperchaotic memristive system are carried out on FPGA, and a discrete time FPGA-based design of the 5D HFWMS is implemented on ModelSim using the RK-4 algorithm. The maximum operating frequency of the designed chaos-based system reaches $147.863 \mathrm{MHz}$. Finally, an active control synchronization of the 5D hyperchaotic system with different structures and a secure chaotic masking communication application are implemented on the platform of Matlab and FPGA, respectively. All these results justify the successful applications of the novel 5D HFWMS in active control synchronization and various chaos-based embedded secure chaotic masking communication systems. Our future work is to study whether the system has multistability, and to apply the system to image encryption and random number generator.

\section{Data Availability}

All data used to support the findings of this study are available from the corresponding author upon request.

\section{Conflicts of Interest}

The authors declare that they have no conflicts of interest.

\section{Acknowledgments}

This work was supported by the National Natural Science Foundation of China under Grants 61504013, 61702052, 61772087, 61674054, and 61901169, Natural Science Foundation of Hunan Province under Grants 2019JJ50648, 2016jj2005, 2017JJ2049, and 2019JJ40190, Scientific Research Fund of Hunan Provincial Education Department under Grant 18A137, and the National Key Research and Development Project under Grant 2018YFE0111200.

\section{References}

[1] S. R. Fulton, "Multigrid solution of non-linear balance equations in meteorology," Communications in Applied Numerical Methods, vol. 8, no. 10, pp. 695-706, 1992.

[2] J. Wu, X. Chen, H. Yao, L. Gao, Y. Chen, and M. Liu, "Nonlinear relationship of hydrological drought responding to meteorological drought and impact of a large reservoir," Journal of Hydrology, vol. 551, pp. 495-507, 2017.

[3] M. Long, F. Peng, and Y. Zhu, "Identifying natural images and computer generated graphics based on binary similarity measures of PRNU," Multimedia Tools and Applications, vol. 78, no. 1, pp. 489-506, 2019.

[4] Y.-S. Huang and Z.-Y. Wang, "Decentralized adaptive fuzzy control for a class of large-scale MIMO nonlinear systems with strong interconnection and its application to automated highway systems," Information Sciences, vol. 274, pp. 210224, 2014.

[5] Q. Xie, X. Wang, Z. Han, Y. Zuo, and M. Tang, "Immersion and invariance control of a class of nonlinear cascaded discrete systems," Neurocomputing, vol. 171, pp. 1661-1665, 2016.

[6] Y.-S. Huang and M. Wu, "Robust decentralized direct adaptive output feedback fuzzy control for a class of largesale nonaffine nonlinear systems," Information Sciences, vol. 181, no. 11, pp. 2392-2404, 2011.

[7] A. M. A. Nasar, B. D. Rogers, A. Revell, and P. K. Stansby, "Flexible slender body fluid interaction: vector-based discrete element method with Eulerian smoothed particle hydrodynamics," Computers \& Fluids, vol. 179, pp. 563-578, 2019.

[8] N. Nguyen, A. Wineman, and A. Waas, "Contact problem of a non-linear viscoelastic spherical membrane enclosing incompressible fluid between two rigid parallel plates," International Journal of Non-Linear Mechanics, vol. 50, pp. 97-108, 2013.

[9] V.-T. Pham, A. Buscarino, L. Fortuna, and M. Frasca, "Autowaves in memristive cellular neural networks," International Journal of Bifurcation and Chaos, vol. 22, no. 8, p. 1230027, 2012.

[10] F. Yu, L. Liu, L. Xiao, K. Li, and S. Cai, "A robust and fixedtime zeroing neural dynamics for computing time-variant nonlinear equation using a novel nonlinear activation function," Neurocomputing, vol. 350, pp. 108-116, 2019.

[11] L. Zhou, F. Tan, F. Yu, and W. Liu, "Cluster synchronization of two-layer nonlinearly coupled multiplex networks with multi-links and time-delays," Neurocomputing, vol. 359, pp. 264-275, 2019.

[12] W. Yao, C. Wang, J. Cao, Y. Sun, and C. Zhou, "Hybrid multisynchronization of coupled multistable memristive neural networks with time delays," Neurocomputing, vol. 363, pp. 281-294, 2019.

[13] V.-T. Pham, S. Vaidyanathan, E. Tlelo-Cuautle, and T. Kapitaniak, "Memory circuit elements: complexity, complex systems, and applications," Complexity, vol. 2019, Article ID 4936123, 4 pages, 2019.

[14] X. Zhang and C. Wang, "A novel multi-attractor period multi-scroll chaotic integrated circuit based on CMOS wide adjustable CCCII," IEEE Access, vol. 7, no. 1, pp. 1633616350, 2019.

[15] R. P. Wu and C. H. Wang, "A new simple chaotic circuit based on memristor," International Journal of Bifurcation and Chaos, vol. 26, no. 9, pp. 1-11, 2016.

[16] R. Condorelli, "Applied nonlinear dynamical system in social science. A nonlinear model for social control system: an application to Italian coercion system," Quality \& Quantity, vol. 47, no. 2, pp. 1173-1198, 2013.

[17] C. Liu and Z.-K. Zhang, "Information spreading on dynamic social networks," Communications in Nonlinear Science and Numerical Simulation, vol. 19, no. 4, pp. 896-904, 2014.

[18] F. Yu, L. Li, Q. Tang, S. Cai, Y. Song, and Q. Xu, "A survey on true random number generators based on chaos," Discrete Dynamics in Nature and Society, vol. 2019, Article ID 2545123, 2019.

[19] O. Reyad and Z. Kotulski, "Statistical analysis of the chaosdriven elliptic curve pseudo-random number generators," Communications in Computer and Information Science, vol. 448, pp. 38-48, 2014.

[20] H. Xu, X. Tong, and X. Meng, "An efficient chaos pseudorandom number generator applied to video encryption," Optik, vol. 127, no. 20, pp. 9305-9319, 2016. 
[21] X. Wang and C. Yu, "Cryptanalysis and improvement on a cryptosystem based on a chaotic map," Computers \& Mathematics with Applications, vol. 57, no. 3, pp. 476-482, 2009.

[22] R. Rhouma and S. Belghith, "Cryptanalysis of a chaos-based cryptosystem on DSP," Communications in Nonlinear Science and Numerical Simulation, vol. 16, no. 2, pp. 876-884, 2011.

[23] Q. Yin and C. Wang, "A new chaotic image encryption scheme using breadth-first search and dynamic diffusion," International Journal of Bifurcation and Chaos, vol. 28, no. 4, p. 1850047, 2018.

[24] Y. Li, C. Wang, and H. Chen, "A hyper-chaos-based image encryption algorithm using pixel-level permutation and bitlevel permutation," Optics and Lasers in Engineering, vol. 90, pp. 238-246, 2017.

[25] G. Cheng, C. Wang, and H. Chen, "A novel color image encryption algorithm based on hyperchaotic system and permutation-diffusion architecture," International Journal of Bifurcation and Chaos, vol. 29, no. 9, Article ID 1950115, 2019.

[26] F. Yu and C. Wang, "Secure communication based on a fourwing chaotic system subject to disturbance inputs," Optik, vol. 125, no. 20, pp. 5920-5925, 2014.

[27] N. Vafamand, S. Khorshidi, and A. Khayatian, "Secure communication for non-ideal channel via robust TS fuzzy observer-based hyperchaotic synchronization," Chaos, Solitons \& Fractals, vol. 112, pp. 116-124, 2018.

[28] L. Zhou, C. Wang, and H. He, "Time-controllable combinatorial inner synchronization and outer synchronization of anti-star networks and its application in secure communication," Communications in Nonlinear Science and Numerical Simulation, vol. 22, no. 1-3, pp. 623-640, 2015.

[29] L. Zhou, F. Tan, and F. Yu, “A robust synchronization-based chaotic secure communication scheme with double-layered and multiple hybrid networks," IEEE Systems Journal, 2019.

[30] F. Yu, L. Gao, K. Gu, B. Yin, Q. Wan, and Z. Zhou, "A fully qualified four-wing four-dimensional autonomous chaotic system and its synchronization," Optik, vol. 131, pp. 79-88, 2017.

[31] F. Yu, P. Li, K. Gu, and B. Yin, "Research progress of multiscroll chaotic oscillators based on current-mode devices," Optik, vol. 127, no. 13, pp. 5486-5490, 2016.

[32] C. H. Wang, H. Xu, and F. Yu, "A novel approach for constructing high-order Chua's circuit with multi-directional multi-scroll chaotic attractors," International Journal of Bifurcation and Chaos, vol. 23, no. 2, Article ID $1350022,2013$.

[33] J. Jin, "Programmable multi-direction fully integrated chaotic oscillator," Microelectronics Journal, vol. 75, pp. 27-34, 2018.

[34] J. Jin and L. Zhao, "Low voltage low power fully integrated chaos generator," Journal of Circuits, Systems and Computers, vol. 27, no. 10, p. 1850155, 2018.

[35] X. Zhang, C. Wang, W. Yao, and H. Lin, "Chaotic system with bondorbital attractors,” Nonlinear Dynamics, vol. 97, no. 4, pp. 2159-2174, 2019.

[36] O. E. Rossler, "An equation for hyperchaos," Physics Letters $A$, vol. 71, no. 2-3, pp. 155-157, 1979.

[37] J. Jin and L. Cui, "Fully integrated memristor and its application on the scroll-controllable hyperchaotic system," Complexity, vol. 2019, Article ID 4106398, 8 pages, 2019.

[38] F. Yu, C. Wang, and H. He, "Grid multiscroll hyperchaotic attractors based on colpitts oscillator mode with controllable grid gradient and scroll numbers," Journal of Applied Research and Technology, vol. 11, no. 3, pp. 371-380, 2013.

[39] C. Volos, J.-O. Maaita, S. Vaidyanathan, V.-T. Pham, I. Stouboulos, and I. Kyprianidis, "A novel four-dimensional hyperchaotic four-wing system with a saddle-focus equilibrium," IEEE Transactions on Circuits and Systems II: Express Briefs, vol. 64, no. 3, pp. 339-343, 2017.

[40] V.-T. Pham, S. Vaidyanathan, C. Volos, S. Jafari, and S. T. Kingni, "A no-equilibrium hyperchaotic system with a cubic nonlinear term," Optik, vol. 127, no. 6, pp. 3259-3265, 2016.

[41] K. Rajagopal, A. J. M. Khalaf, Z. Wei, V.-T. Pham, A. Alsaedi, and T. Hayat, "Hyperchaos and coexisting attractors in a modified van der Pol-duffing oscillator," International Journal of Bifurcation and Chaos, vol. 29, no. 5, Article ID 1950067, 2019.

[42] X. Zhang and C. Wang, "Multiscroll hyperchaotic system with hidden attractors and its circuit implementation," International Journal of Bifurcation and Chaos, vol. 29, no. 9, Article ID 1950117, 2019.

[43] C. X. Zhang and S. M. Yu, "A novel methodology for constructing a multi-wing chaotic and hyperchaotic system with a unified step function switching control," Chinese Physics B, vol. 25, no. 5, Article ID 050503, 2016.

[44] C. Zhang, "Theoretical design approach of four-dimensional piecewise-linear multi-wing hyperchaotic differential dynamic system," Optik, vol. 127, no. 11, pp. 4575-4580, 2016.

[45] C. Zhang, "Mirror symmetry multi-wing attractors generated from a novel four-dimensional hyperchaotic system," Optik, vol. 127, no. 5, pp. 2924-2930, 2016.

[46] A. Zarei, "Complex dynamics in a 5-D hyper-chaotic attractor with four-wing, one equilibrium and multiple chaotic attractors," Nonlinear Dynamics, vol. 81, no. 1-2, pp. 585-605, 2015.

[47] L. Chua, "Memristor-the missing circuit element," IEEE Transactions on Circuit Theory, vol. 18, no. 5, pp. 507-519, 1971.

[48] D. B. Strukov, G. S. Snider, D. R. Stewart, and R. S. Williams, "The missing memristor found," Nature, vol. 453, no. 7191, pp. 80-83, 2008.

[49] S. P. Wang, C. L. He, J. Tang et al., "Electronic synapses based on ultrathin quasi-two-dimensional gallium oxide memristor," Chinese Physics B, vol. 28, no. 1, pp. 183-188, 2019.

[50] P. Prakash, J. P. Singh, and B. K. Roy, "Fractional-order memristor-based chaotic system with a stable equilibrium point, its fractional-order PI-based sliding mode control and switching synchronisation," Pramana, vol. 92, no. 2, 2019.

[51] V.-T. Pham, A. Buscarino, L. Fortuna, and M. Frasca, "Simple memristive time-delay chaotic systems," International Journal of Bifurcation and Chaos, vol. 23, no. 4, Article ID 1350073, 2013.

[52] C. K. Volos, A. Akgul, V. T. Pham et al., "Antimonotonicity, crisis and multiple attractors in a simple memristive circuit," Journal of Circuits, Systems and Computers, vol. 27, no. 2, pp. 80-83, 2018.

[53] C. Wang, X. Liu, and H. Xia, "Multi-piecewise quadratic nonlinearity memristor and its $2 \mathrm{~N}$-scroll and $2 \mathrm{~N}+1$-scroll chaotic attractors system," Chaos, vol. 27, no. 3, Article ID 033114, 2017.

[54] J. Ma, Z. Chen, Z. Wang, and Q. Zhang, "A four-wing hyperchaotic attractor generated from a 4-D memristive system with a line equilibrium," Nonlinear Dynamics, vol. 81, no. 3, pp. 1275-1288, 2015. 
[55] L. Zhou, C. Wang, and L. Zhou, "A novel no-equilibrium hyperchaotic multi-wing system via introducing memristor," International Journal of Circuit Theory and Applications, vol. 46, no. 1, pp. 84-98, 2018.

[56] L. Zhou, C. Wang, and L. Zhou, "Generating four-wing hyperchaotic attractor and two-wing, three-wing, and fourwing chaotic attractors in $4 \mathrm{D}$ memristive system," International Journal of Bifurcation and Chaos, vol. 27, no. 2, p. 1750027, 2017.

[57] L. Zhou, C. Wang, and L. Zhou, "Generating hyperchaotic multi-wing attractor in a $4 \mathrm{D}$ memristive circuit," Nonlinear Dynamics, vol. 85, no. 4, pp. 2653-2663, 2016.

[58] F. Yu, Q. Tang, W. Wang, and H. Wu, “A 2.7 GHz low-phasenoise LC-QVCO using the gate-modulated coupling technique," Wireless Personal Communications, vol. 86, no. 2, pp. 671-681, 2016.

[59] F. Yu, L. Gao, L. Liu, S. Qian, S. Cai, and Y. Song, "A 1 V, $0.53 \mathrm{~ns}, 59 \mu \mathrm{W}$ current comparator using standard $0.18 \mu \mathrm{m}$ CMOS technology," Wireless Personal Communications, 2019.

[60] F. Yu, "A low-voltage and low-power 3-GHz CMOS LC VCO for S-band wireless applications," Wireless Personal Communications, vol. 78, no. 2, pp. 905-914, 2014.

[61] S. He, K. Sun, and H. Wang, "Complexity analysis and DSP implementation of the fractional-order Lorenz hyperchaotic system," Entropy, vol. 17, no. 12, pp. 8299-8311, 2015.

[62] Z. Xiao-Hong and Z. Zhi-Guang, "Digital circuit design and simulation of a new time-delay hyperchaotic system," Communications in Computer and Information Science, vol. 234, pp. 411-417, 2011.

[63] M. Tuna, M. Alçın, İ. Koyuncu, C. B. Fidan, and İ. Pehlivan, "High speed FPGA-based chaotic oscillator design," Microprocessors and Microsystems, vol. 66, pp. 72-80, 2019.

[64] A. Ahmadi, K. Rajagopal, F. E. Alsaadi, V.-T. Pham, F. E. Alsaadi, and S. Jafari, "A novel 5D chaotic system with extreme multi-stability and a line of equilibrium and its engineering applications: circuit design and FPGA implementation," Iranian Journal of Science and Technology, Transactions of Electrical Engineering, 2019.

[65] Y. M. Xu, L. D. Wang, and S. K. Duan, "A memristor-based chaotic system and its field programmable gate array implementation," Acta Physica Sinica, vol. 65, no. 12, p. 120503,2016

[66] E. Tlelo-Cuautle, A. D. Pano-Azucena, J. J. Rangel-Magdaleno, V. H. Carbajal-Gomez, and G. Rodriguez-Gomez, "Generating a 50 -scroll chaotic attractor at $66 \mathrm{MHz}$ by using FPGAs," Nonlinear Dynamics, vol. 85, no. 4, pp. 2143-2157, 2016.

[67] J.-L. Zhang, W.-Z. Wang, X.-W. Wang, and Z.-H. Xia, "Enhancing security of FPGA-based embedded systems with combinational logic binding," Journal of Computer Science and Technology, vol. 32, no. 2, pp. 329-339, 2017.

[68] L. Yong, "Lag synchronization of autocatalytic chemical reaction in continuous stirred tank reactors," Acta Physica Sinica, vol. 57, no. 10, pp. 6099-6102, 2008.

[69] X. Wu, C. Bai, and H. Kan, "A new color image cryptosystem via hyperchaos synchronization," Communications in Nonlinear Science and Numerical Simulation, vol. 19, no. 6, pp. 1884-1897, 2014.

[70] M. Rafikov and J. M. Balthazar, "On control and synchronization in chaotic and hyperchaotic systems via linear feedback control," Communications in Nonlinear Science and Numerical Simulation, vol. 13, no. 7, pp. 1246-1255, 2008.
[71] C.-L. Li and L. Wu, "Sliding mode control for synchronization of fractional permanent magnet synchronous motors with finite time," Optik, vol. 127, no. 6, pp. 3329-3332, 2016.

[72] F. Yu and Y. Song, "Complete switched generalized function projective synchronization of a class of hyperchaotic systems with unknown parameters and disturbance inputs," Journal of Dynamic Systems, Measurement, and Control, vol. 136, no. 1, Article ID 014505, 2013.

[73] F. Yu, C. Wang, Q. Wan, and Y. Hu, "Complete switched modified function projective synchronization of a five-term chaotic system with uncertain parameters and disturbances," Pramana, vol. 80, no. 2, pp. 223-235, 2013.

[74] F. Yu, C. H. Wang, Y. Hu, and J. W. Yin, "Antisynchronization of a novel hyperchaotic system with parameter mismatch and external disturbances," Pramana, vol. 79, no. 1, pp. 81-93, 2012.

[75] J. Yu, J. Lei, and L. Wang, "Backstepping synchronization of chaotic system based on equivalent transfer function method," Optik, vol. 130, pp. 900-913, 2017.

[76] Y.-Y. Huang, Y.-H. Wang, and Y. Zhang, "Shape synchronization of drive-response for a class of two-dimensional chaotic systems via continuous controllers," Nonlinear Dynamics, vol. 78, no. 4, pp. 2331-2340, 2014.

[77] Y. Huang, Y. Wang, H. Chen, and S. Zhang, "Shape synchronization control for three-dimensional chaotic systems," Chaos, Solitons \& Fractals, vol. 87, pp. 136-145, 2016.

[78] S. Çiçek, A. Ferikoğlu, and İ. Pehlivan, "A new 3D chaotic system: dynamical analysis, electronic circuit design, active control synchronization and chaotic masking communication application," Optik, vol. 127, no. 8, pp. 4024-4030, 2016.

[79] C. Huang and J. Cao, "Active control strategy for synchronization and anti-synchronization of a fractional chaotic financial system," Physica A: Statistical Mechanics and its Applications, vol. 473, pp. 262-275, 2017.

[80] I. Ahmad, A. B. I. Saaban, A. B. Saaban, A. B. Ibrahim, M. Shahzad, and N. Naveed, "The synchronization of chaotic systems with different dimensions by a robust generalized active control," Optik, vol. 127, no. 11, pp. 4859-4871, 2016.

[81] K. Xie, X. Ning, X. Wang et al., "An efficient privacy-preserving compressive data gathering scheme in WSNs," Information Sciences, vol. 390, no. 2, pp. 702-715, 2016.

[82] M. Long, F. Peng, and H.-Y. Li, "Separable reversible data hiding and encryption for HEVC video," Journal of RealTime Image Processing, vol. 14, no. 1, pp. 171-182, 2018.

[83] K. Gu, K. Wang, and L. Yang, "Traceable attribute-based signature," Journal of Information Security and Applications, vol. 49, pp. 1-16, 2019.

[84] K. Gu, N. Wu, B. Yin, and W. Jia, "Secure data sequence query framework based on multiple fogs," IEEE Transactions on Emerging Topics in Computing, 2019.

[85] K. Gu, N. Wu, B. Yin, and W. Jia, "Secure data query framework for cloud and fog computing," IEEE Transactions on Network and Service Management, 2019.

[86] K. Gu, W. Jia, G. Wang et al., "Efficient and secure attributebased signature for monotone predicates," Acta Informatica, vol. 54, pp. 521-541, 2017.

[87] Z. Xia, Z. Fang, F. Zou, J. Wang, and A. K. Sangaiah, "Research on defensive strategy of real-time price attack based on multiperson zero-determinant," Security and Communication Networks, vol. 2019, pp. 1-13, 2019.

[88] S. He, W. Zeng, K. Xie et al., "PPNC: privacy preserving scheme for random linear network coding in smart grid," KSII Transactions on Internet and Information Systems, vol. 11, pp. 1510-1533, 2017. 
[89] L. Xiang, X. Shen, J. Qin, and W. Hao, "Discrete multi-graph hashing for large-scale visual search," Neural Processing Letters, vol. 49, no. 3, pp. 1055-1069, 2019.

[90] L. Xiang, Y. Li, W. Hao et al., "Reversible natural Language watermarking using synonym substitution and arithmetic coding," CMC: Computers, Materials and Continua, vol. 55, no. 3, pp. 541-559, 2018

[91] K. Gu, L. Yang, Y. Wang, and S. Wen, "Traceable identitybased group signature," RAIRO-Theoretical Informatics and Applications, vol. 50, no. 3, pp. 193-226, 2016.

[92] Z. Sun, L. Si, Z. Shang, and J. Lei, "Finite-time synchronization of chaotic PMSM systems for secure communication and parameters identification," Optik, vol. 157, pp. 43-55, 2018.

[93] W. Wang, X. Jia, X. Luo, J. Kurths, and M. Yuan, "Fixed-time synchronization control of memristive MAM neural networks with mixed delays and application in chaotic secure communication," Chaos, Solitons \& Fractals, vol. 126, pp. 85-96, 2019.

[94] S. Khorashadizadeh and M.-H. Majidi, "Chaos synchronization using the Fourier series expansion with application to secure communications," AEU-International Journal of Electronics and Communications, vol. 82, pp. 37-44, 2017.

[95] M. F. Hassan and M. Hammuda, "A new approach for constrained chaos synchronization with application to secure data communication," Journal of the Franklin Institute, vol. 356, no. 12, pp. 6697-6723, 2019.

[96] M. Xu, W. Pan, and L. Zhang, "Secure remote synchronization and secure key distribution in electro-optic networks revealed by symmetries," Optics Communications, vol. 418, pp. 41-45, 2018.

[97] M. E. Sahin, Z. G. Cam Taskiran, H. Guler, and S. E. Hamamci, "Simulation and implementation of memristive chaotic system and its application for communication systems," Sensors and Actuators A: Physical, vol. 290, pp. 107-118, 2019.

[98] R. Ramesh Babu and R. Karthikeyan, "Adaptive synchronization of novel chaotic system and its FPGA implementation," in Proceedings of the 2015 International Conference on Smart Technologies and Management for Computing, Communication, Controls, Energy and Materials (ICSTM), pp. 449-454, Chennai, India, May 2015.

[99] T. Tami, T. Messaoudene, A. Ferdjouni et al., "Chaos secure communication' implementation in FPGA," in Proceedings of the 2018 International Conference on Applied Smart Systems (ICASS), Medea, Algeria, November 2018.

[100] K. Rajagopal, A. Durdu, S. Jafari, Y. Uyaroglu, A. Karthikeyan, and A. Akgul, "Multiscroll chaotic system with sigmoid nonlinearity and its fractional order form with synchronization application," International Journal of NonLinear Mechanics, vol. 116, pp. 262-272, 2019.

[101] S. He, K. Sun, and S. Banerjee, "Dynamical properties and complexity in fractional-order diffusionless Lorenz system," European Physical Journal Plus, vol. 131, p. 254, 2016.

[102] S. He, K. Sun, X. Mei et al., "Numerical analysis of fractionalorder chaotic system based on conformable fractional-order derivative," European Physical Journal Plus, vol. 132, p. 36, 2017.

[103] M. Tuna and C. B. Fidan, "Electronic circuit design, implementation and FPGA-based realization of a new 3D chaotic system with single equilibrium point," Optik, vol. 127, no. 24, pp. 11786-11799, 2016.

[104] Q. Yang and M. Bai, "A new 5D hyperchaotic system based on modified generalized Lorenz system," Nonlinear Dynamics, vol. 88, no. 1, pp. 189-221, 2017. 


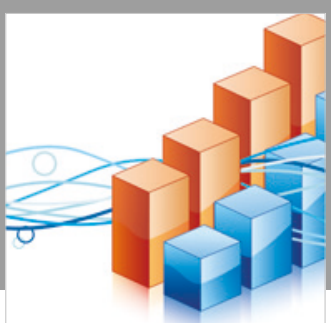

Advances in

Operations Research

\section{-n-m}
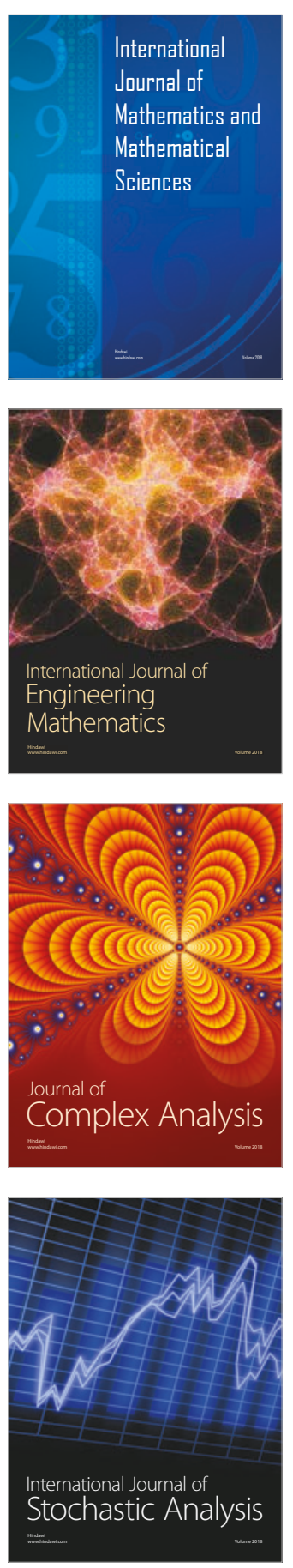
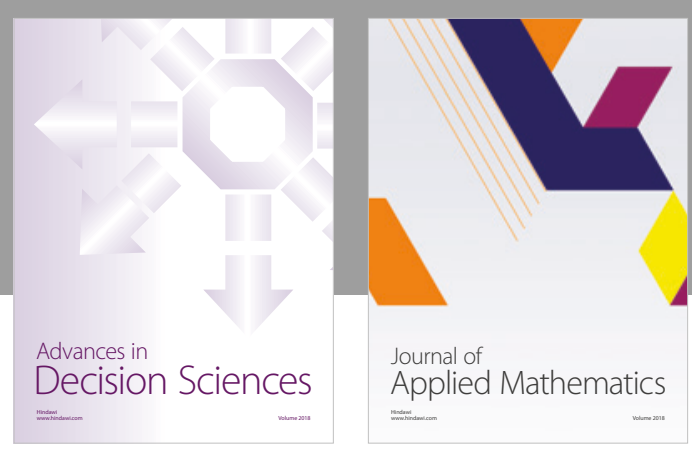

Journal of

Applied Mathematics
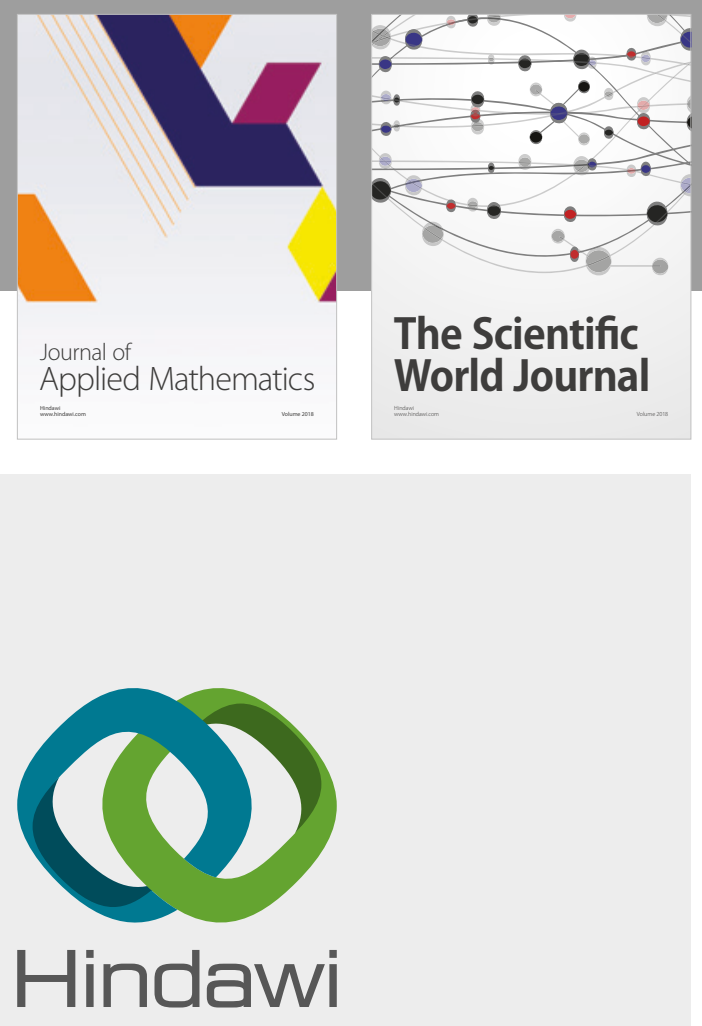

Submit your manuscripts at

www.hindawi.com

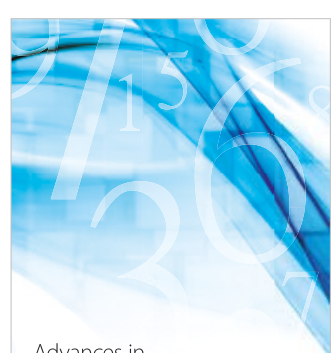

Advances in
Numerical Analysis
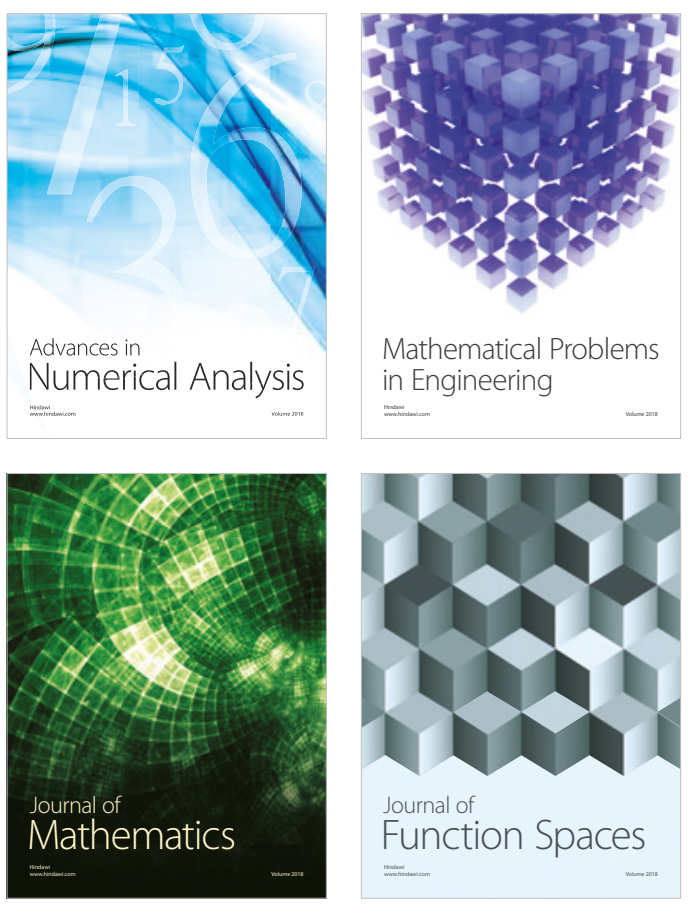

Mathematical Problems in Engineering

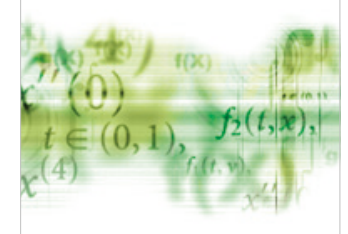

International Journal of

Differential Equations

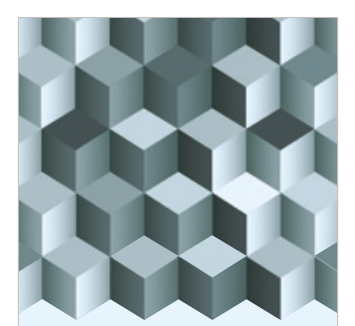

Journal of

Function Spaces
The Scientific

World Journal

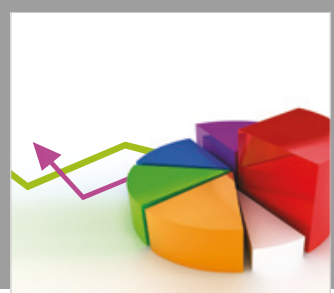

Journal of

Probability and Statistics
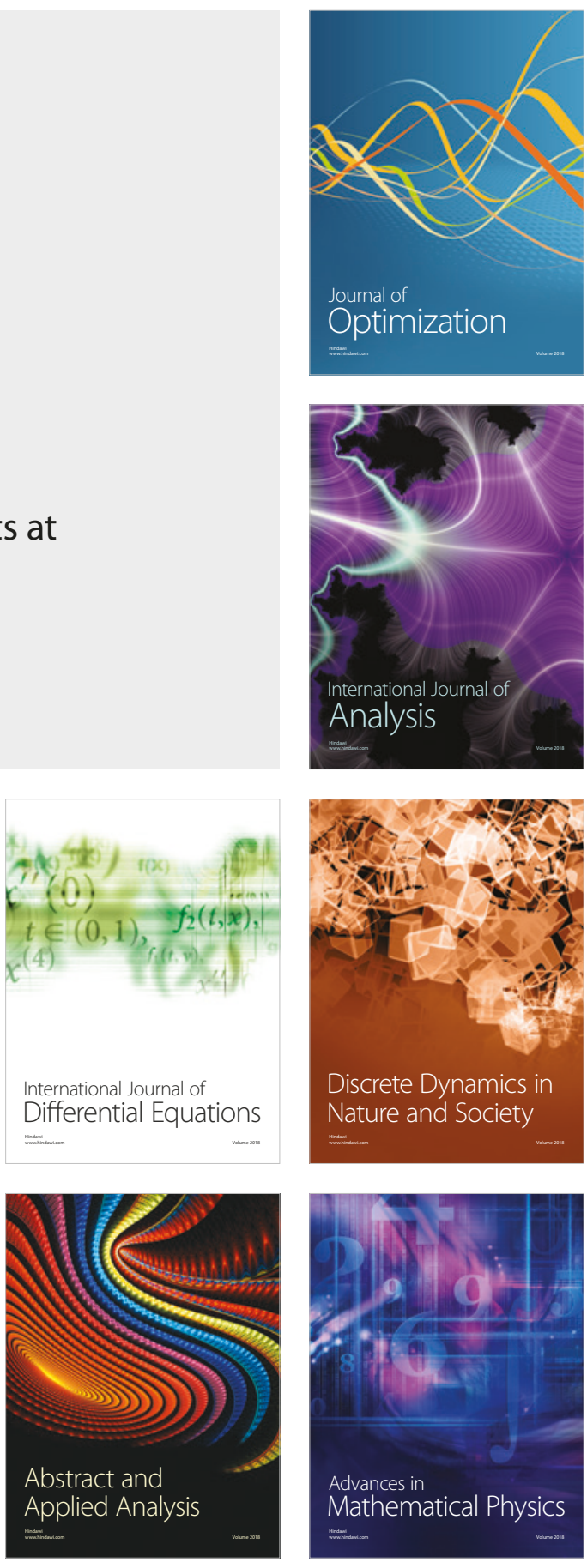